\title{
Guided Folding of Life's Proteins in Integrate Cells with Holographic Memory and GM-Biophysical Steering
}

\author{
Dirk K. F. Meijer1*, Hans J. H. Geesink ${ }^{2}$ \\ ${ }^{1}$ University of Groningen, Groningen, The Netherlands \\ ${ }^{2}$ Biophysics Group, Loon op Zand, The Netherlands \\ Email: ^meij6076@planet.nl
}

How to cite this paper: Meijer, D.K.F. and Geesink, H.J.H. (2018) Guided Folding of Life's Proteins in Integrate Cells with Holographic Memory and GM-Biophysical Steering. Open Journal of Biophysics, 8, 117-154.

https://doi.org/10.4236/ojbiphy.2018.83010

Received: April 26, 2018

Accepted: July 14, 2018

Published: July 17, 2018

Copyright $\odot 2018$ by authors and Scientific Research Publishing Inc. This work is licensed under the Creative Commons Attribution International License (CC BY 4.0).

http://creativecommons.org/licenses/by/4.0/

\begin{abstract}
The current geometric and thermodynamic approaches in protein folding studies do not provide a definite solution to understanding mechanisms of folding of biological proteins. A major problem is that the protein is first synthesized as a linear molecule that subsequently must reach its native configuration in an extremely short time. Hydrophobicity-hydrophilicity models and random search mechanism cannot explain folding to the 3-D functional form in less than 1 second, as it occurs in the intact cell. We propose an integral approach, based on the embedding of proteins in the whole cellular context under the postulate: a life protein is never alone. In this concept the protein molecule is influenced by various long and short distance force fields of nature such as coherent electromagnetic waves and zero-point energy. In particular, the role of solitons is reviewed in relation to a novel GM-scale biophysical principle, revealed by us. This recent finding of a set of discrete EM frequency bands, that either promote or endanger life conditions, could be a key in further studies directed at the morphogenetic aspects of protein folding in a biological evolutionary context. In addition, an alternative hypothesis is presented in which each individual cell may store integral 3-D information holographically at the virtual border of a 4-D hypersphere that surrounds each living cell, providing a field receptive memory structure that is instrumental in guiding the folding process towards coherently oscillating protein networks that are crucial for cell survival.
\end{abstract}

\section{Keywords}

Protein Folding, Coherent Wave Pattern, Electromagnetic Fields, Solitons, Harmonics, Cellular Automata, Quantum Mechanics 


\section{Introduction}

The issue of protein folding is often considered using two different approaches that can be called "biophysical" and "geometrical" [1]. The "biophysical" approach uses concepts such as Gibbs free energy, entropy, and temperature to study protein folding. Simulations of folding are based on statistical physics. In the "geometrical" approach, folding is considered geometrically, as a part of the broader context of the folding of figures of different topologies. A major problem (the so called Levinthal's paradox) is that the protein is first synthesized as a linear molecule that subsequently must reach its native configuration in a short time (on the order of seconds or less, Figure 1). Importantly, the protein can only perform its functions in this (often single) configuration. The problem, however, is that the number of possible conformational states is exponentially large for a long protein molecule. Despite the almost 30 years of attempts to resolve this paradox, a definite solution has not yet been found. In particular, computational biology has shown that the problem of folding based on H-P

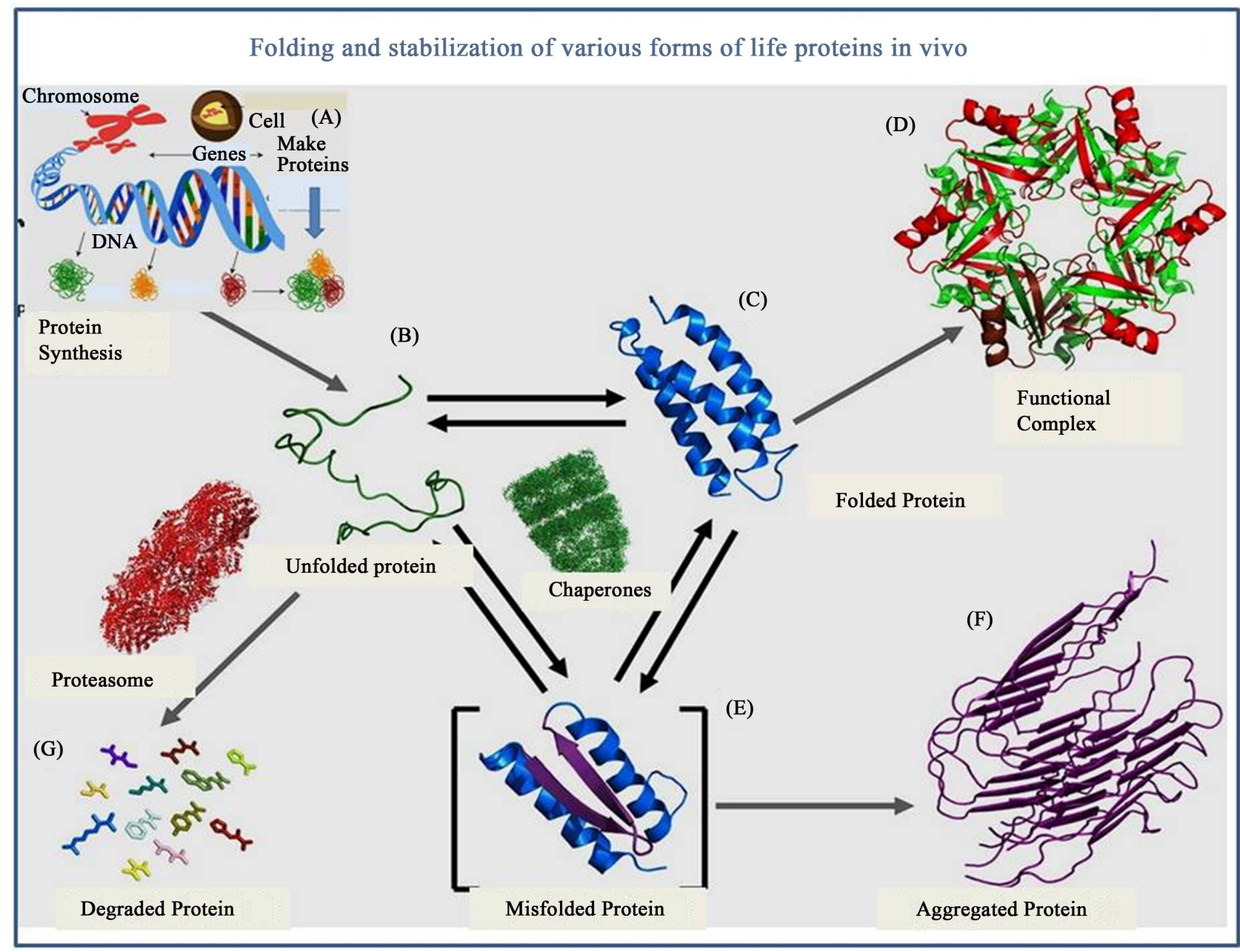

Figure 1. Protein folding in the cell; (A): Ribosomal protein synthesis; (B): Native state; (C): Folded functional conformation; (D): Functional complex; (E): Misfolded form; (F): Toxic aggregate; (G): Degradation products; (modified from Munoz and Cerminara, 2016). 
(hydrophobic-hydrophilic) model, is extremely complex (i.e., generally requires an enormous number of steps [2]. Levinthal concluded rightfully that a random search can only be performed in an unrealistic timeframe of billions of years [1]. Thus two major questions remain: what are the actual folding mechanisms and how can this ultra-rapid process be realized in the whole, intact, cell structure?

Martinez noted that the current folding models had weak predictive ability [3]. Natural proteins consist of 100 to 500 building blocks (alpha-amino-acids), but a random search mechanism cannot provide real-time folding on the order of $1 \mathrm{sec}$. for these proteins. In the framework of a funnel-like energy landscape, the author considers a simple kinetic model of protein folding. However, this kinetic model assumes that once a domain reaches the correct state, it stays there. However, it remains unclear how the domain "knows" that it reached the correct state. After all, an intermediate state is not the minimum energy a state-the minimum is only expected for the whole structure. In the current geometric and thermodynamic approaches in protein folding mechanisms, impressive progress was made, as for instance exemplified by recent papers [4] [5], yet such bottom up approaches fail to provide a satisfactory and generally predictive solution.

Munoz and Cerminara recently reviewed current generation of thermodynamic methods to map ultrafast folding landscapes, interaction networks and various mechanisms at nearly atomic resolution and concluded that folding is tightly coupled to a large number of processes in what they call the protein life cycle (see Figure 1), but also stated that there is a deep divide between what theory and simulations predicted and what experiments showed [6]. Bédard et al. earlier pointed out that folding should be seen as a heterogeneous process with a spectrum of intermediates that is not so much based on independent and unrelated pathways [2]. Rather, it seems governed by the same step-wise pathway that depends on the cooperative interaction with, so called, foldon units of the target native protein, that is, in a predetermined pathway sequence that however is subject to misfolding errors. All this may be taken as an illustration of the complexity of the folding process in vitro, not to speak of the multi-factorial situation in the intact cell.

\section{Alternatives for Classical Approaches}

The present study aims at creating a systematic overview of a wide spectrum of EM frequencies that may influence protein folding, in order to unravel the mechanisms by which proteins obtain their functional conformation as parts of integral vibratory networks for inter- and intra-cellular communication.

In order to present a complimentary view to existing theories, a versatile approach to the problem is presented, based on the embedding of proteins in an integral cellular context. This includes, apart from the known genome and proteome modalities, a well defined "electrome" aspect [7]. We consider the protein molecule as being influenced by the various long and short distance 
force fields of nature such as gravity, electromagnetism, and zero-point energy, in addition to intrinsic vibratory states of macromolecules that generate coherent excitations in the cell. By defining the condition in which the protein is finally shaped in its 3-D functional configuration in more detail, first of all the interaction of the polypeptide structure with interfacial, potentially structured water dipole molecules and inorganic ions should be taken into account (Figure 2). In addition the (partly)-folded polypeptide chain may undergo functional quantum entanglement with (nearly)-identical proteins, specific substrate molecules and chaperones [8] [9]. Special reference should also be given to the permanent influence of magneto-reception, due to the exposure to external and internal electromagnetic fields, that exhibit discrete radiation frequencies and have been shown by us to influence life conditions [10] [11] [12]. These morphogenetic mechanisms can be mediated by phonon/soliton excitations that, according to abundant literature, lead to coherent vibrational domains in cells that also can take the form of wave/particle condensates and non-local multi-boson fields

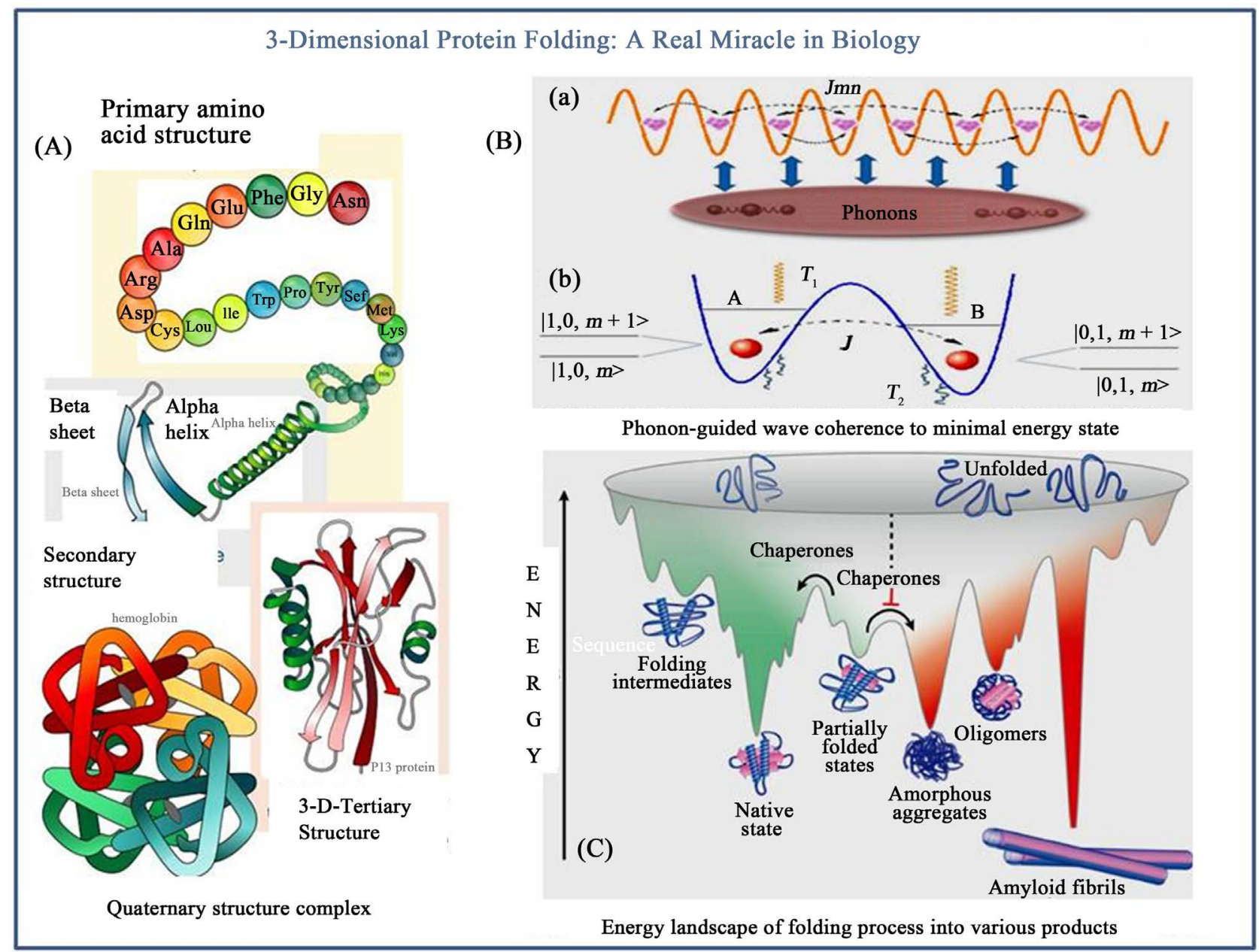

Figure 2. (A): Protein folding from primary to quaternary structure; (B): Influence of phonons on wave coherence, leading to long-lived oscillations through energy dissipation (b) and protection to environmental noise (modified from Zhang and Wang, 2016) (C): Energy landscape with intramolecular (left) and intermolecular aspects (right), producing various endproducts. 
(reviewed by Belyaev [13], Brizhik [14], Cifra [15], Cosic et al. [16], Hammerschlag et al. [17], Levin [18], Muehsam [19], Pokorny [20], Rakovic [21], Tuszynski et al. $[22])$.

In this framework an alternative hypothesis is presented in which we envision a fractal toroidal operator structure to collect and integrate the abovementioned wave information of cells, including their proteins and stores it in a holographic manner on a 3-D virtual screen at the border of a 4-D hypersphere that surrounds each individual cell. This nested torus geometry is fractal at the various organizational levels of the living cell and also provides aspects of error-correction and quality control of the protein folding process. The torus geometry also potentially opens a fourth spatial dimension with a symmetric time aspect, in which the particular protein can be directed to a final functional configuration in the context of the integral cell and whole cell context. This theory finds support in the earlier used computational neural network models, in which integral forward processing of information in a neuronal network, leads to a growing addressable holographic memory space [23]. The global and local excitation mechanisms and the presence of various cytoplasmic factors within the cell, clearly imply that "a life protein molecule is never alone", as depicted in Table 1 .

\section{Long Range Coherence of Vibrational Mechanisms in Protein Folding}

Biomolecular systems exhibit broad vibrational spectral features in the terahertz regime, corresponding to functionally relevant, global, and sub global collective modes [24]. This idea is supported by terahertz absorption spectroscopy, molecular dynamics simulations and normal mode analysis (NMA) that demonstrated the broad terahertz absorption of various proteins in partially solvated

Table 1. A protein molecule in the organism is never alone.

- Proteins bind to transport proteins facilitating nucleus to cytoplasm transport.

- Proteins can be associated with cytoplasmic receptor and chaperone proteins.

- Protein molecules are imbedded in dipole water molecules that can take a clustered form.

- Protein molecules undergo high-frequency spatial changes inducing vibratory conditions.

- Unfolded (parts of) proteins adsorb (hydrated) cytoplasmic alkali cations like $\mathrm{K}^{+}$and anions.

- Cytoplasmic proteins bind organic compounds such as ATP and fatty acids.

- Proteins are exposed to various force fields: electromagnetism, gravity, dark energy and zero-point energy.

- Influence of force fields on protein structure is collected and integrated by $4 \mathrm{D}$ toroidal geometry.

- Formation of functional 3-D proteins requires a combined genomic/electromic machinery.

- Protein molecules undergo conformational changes that induce vibratory states.

- Different protein molecules can show mutual specific coherent resonance patterns.

- Proteins can communicate in functional networks through their resonant states.

- Protein morphology is influenced by external electromagnetic and geo-magnetic fields.

- Protein molecule folding is influenced by coherent macromolecular vibration domains in the cell.

- Protein geometry can be modeled using space-time with 4 spatial dimensions.

- Protein structure can, in principle, be modified by quantum entanglement as a long-distant aspect. 
conditions, as well as in aqueous environments (Figure 2) Terahertz vibrational properties of biomolecules resulted in the accessibility of the so-called "terahertz gap" in a water environment. Fröhlich pointed out that energy provided to terahertz modes of a protein may not be completely thermalized but can be redistributed via the nonlinear processes towards specific low-frequency modes of vibration of the biomolecular structure. Fröhlich's description was based upon on a quantum Hamiltonian, also taking into account the dynamics of phonons of each normal mode of vibration, ruled by linear and nonlinear interactions with a thermal environment (e.g., the cell cytoplasm) as well as an external source of energy (biochemical reactions, ATP hydrolysis, endogenous electromagnetic fields). It can be shown that the system of normal modes will rapidly achieve a steady state, characterized by a non-thermal energy distribution, where most of the phonons will adopt modes of lowest frequencies. Applied to biological systems, this effect is equivalent to having the subsystems of a biomolecular structure oscillate cooperatively over long distances at specific frequencies. As emphasized by Reimers et al., if a coherent effect similar to Fröhlich's effect would be experimentally validated, this could lead to numerous applications in physics, biology, and medicine [25].

Similar collective properties of a system such as lasing, superconductivity, and Bose-Einstein condensation have been suggested. Nowadays, several authors have reported the existence of long-lived excited low-frequency modes in protein structures. For example, Lundholm et al. used combined terahertz techniques with a highly sensitive X-ray crystallographic method to visualize the low-frequency vibrational modes in the crystal structure of hen-egg white lysozyme [26]. This study was later substantiated in near-field $\mathrm{THz}$ absorption spectroscopic studies on in proteins in watery solution [27]. Such studies indicate that $0.4 \mathrm{THz}$ electromagnetic radiation induces non-thermal changes in electron density. In particular, a local increase of electron density in a long alpha-helix motif was observed consistent with a subtle longitudinal compression of the helix at a micro- to milli-second lifetime.

Also, microtubules and cell membranes-for which the hydrolysis of adenosine triphosphate (ATP) or guanosine triphosphate (GTP) provides sources of energy supply-would also be good candidates for Fröhlich's effect. Pokorny, experimentally observed a strong excitation in the spectrum of vibration of microtubules localized in the $10 \mathrm{MHz}$ range that could also be a further evidence of Fröhlich condensation at lower frequencies [20]. Such interactions are thought to play an important role in biomolecular organization, since unlike electrostatic forces, their range could extend far beyond the Debye length. Such interactions occur only when specific modes of the coupled system are strongly excited. Devices such as the Fluorescent Correlation Spectroscopy enable the detection of long-distance interactions between biomolecules in solution by measuring their diffusion coefficient as a function of their concentration [28].

Goushcha explained the idea behind the Davydov's soliton as follows: solitons can be stabilized through the hydrolysis of adenosine triphosphate (ATP) that 
creates excited vibrational states in the peptide group, allowing vibrational excitation to propagate to the neighboring groups due to dipole interaction between groups [29].

The excitation interacts also with the hydrogen bond, creating a local deformation that is coupled (self-trapped) to the vibrational excitation through a feedback mechanism. If the feedback is strong enough, a new, non-dissipative state associated with vibrational excitation and hydrogen bond distortion will be created and will propagate coherently along the peptide chain.

Conventional theories cannot explain how the folding protein chooses a single stable structure among billions of others, nor how freely movable ion channel proteins organize themselves into metastable periodic patterns. Rather, such structures may finally evolve into functional clusters, through steering mechanisms of non-thermal biological effects of weak electromagnetic fields with a quantum energy far below the average energy of thermal fluctuations etc. The currently most successful models consider the protein folding as a two-state first-order phase transition triggered by a spontaneous nucleation and progressing along the energy funnel-type landscape by choosing thermodynamically most favorable barrier-crossing steps, facilitated by favorable conformational states. The funnel landscape in the proposed models emerges only for those amino acid sequences for which there is a structure in which nearly all the various interactions are simultaneously minimized; i.e., native proteins are only minimally frustrated (see later).

The self-organization of the protein folding process is supposed to augment itself by enhancing the stability of the core against large-scale motions that would unfold the protein and stabilizing the native state through hydrophobic interaction that drives the overall collapse of the chain robustness of the native structure against mutations, etc. Yet, a unifying concept that combines the basic features governing self-organization of proteins into complex three-dimensional structures both in vitro and in vivo is still lacking. This may be attributed to a complexity of the folding process that involves a very large number of interactions (with structural feedback), allowing calculation of only relatively small native structures and other critical limitations. Current studies are focused on numerous as yet unresolved questions, such as unfolding-refolding reactions and the role of hidden intermediates such as water dynamics in guiding the chain movements.

Importantly, it should be mentioned here that, very likely, proteins do not adopt their native structure in-vitro conditions, but rather require a complete cellular machinery to correctly fold in vivo (see Rost, 2003, for many relevant references [30]).

An interesting evolutionary concept on protein folding was put forward by Wolynes in which the folding process is modeled in a energy landscape funnel, and related to the, so called, minimal frustration theory [5]. The author pictures the becoming process of a single functional life protein as an interplay between funnel-shaped information spaces of sequence versus configuration entropies 
and thus of an ongoing superposition of information states with regard to protein composition and its final functional 3-D form. In these processes the protein passes several energy barriers that, to various degrees, can frustrate the temporal aspect of the folding. We argue in the following sections, that such frustration modalities, that in fact represent "conflicts" in choice of favorable interactions in the folding process, could be "helped" by local coherent vibrational solitonic states.

\section{The Cellular Protein Electrome as Influenced by Discrete EM Frequency Bands}

As mentioned above, living cells contain an oscillating macromolecular apparatus, tentatively called the cellular electrome [7] that can be excited by internal as well as by external electromagnetic fields. Through a process of resonance, the various geometry features can become expressed in life systems as coherent vibratory patterns that somehow influence the functional structure and metabolism of the exposed cell systems. The particular internal oscillations may mirror typical mathematical eigenfrequencies of the various external fields over a broad range of frequencies. This resonant coherency is analogue to the principles of Fröhlich-Bose-Einstein condensates as initially postulated by Fröhlich (1975) [31] and later elaborated in a soliton model by Davydov (1977) [32] [33] and Pang et al. (2006) [34] and, as mentioned above, more recently identified in cells by spectrometric experimentation [26].

The Fröhlich/Davydov concept has been elaborated and further improved by Pang taking into account that solitons can be largely stabilized, and their life-time increased due to mutual interaction of the particles with lattice vibrations [35]. Consequently the total state of the system has been expressed in three different Hamiltonians. Due to these extensions, the solitons obtain life-times that are more compatible with the ruling biological conditions. This was expressed in Hamiltonians on quasi-coherent two-quantum state wave functions [34].

It is of interest that, for example, coherent longitudinal vibration of micro-tubular proteins may induce electromagnetic fields in the cell [15]. Thus externally applied electromagnetic fields with discrete frequencies may, through resonant mechanisms, interact with endogenous cellular fields that are produced by coherently oscillating macromolecules of the cell, that in concert provide the electrome aspect of the cell [7].

\section{Potential Influence of Electromagnetic Fields in Life Systems}

An extensive meta-analysis of 500 published biological/medical studies was earlier performed [10] [11], in which living material (tissues, cells, and whole animals) were exposed to external electromagnetic fields, employing a wide spectrum of frequencies. In these studies the various discrete electromagnetic frequency bands were studied as to their life-sustaining, as opposed to detrimental 
actions. After collecting and scrutinizing these data, a striking pattern of frequency bands was revealed. The particular bands, representing soliton frequency zones, showed a discrete distribution pattern, plotted on an acoustic scale, in which the separation of the bands was complete and statistically highly significant. The life stabilizing electromagnetic frequency patterns of this "Life algorithm principle" could be modelled as spiral information trajectories using a toroidal geometry, as earlier shown in music theory [11]. The potential EM excitation/resonance of cell components via the detected discrete EM frequency bands were in line with almost identical eigenvalues, calculated by Ritz [36], of the sound-induced geometric/fractal patterns produced by sound excitation of vibrating membranes/plates as reported by Chladny, already in 1817 [37] and three subsequent follow-up studies of others from 1950 up to the present [11].

The actual nature of living cells and potential life supporting effects due to endogenous and exogenous EM field exposition can encompass the following mechanisms:

1) Electromagnetic fields directly influence trans-membrane ion gradients such as for $\mathrm{Ca}^{2+}$, that are pivotal in cellular signalling [11] [38] [39] or perturb collective movements of extracellular (interstitial) ions that can be seen as an active plasma-like workspace [40].

2) Specific EM frequencies can cause vibrational resonance with macromolecules in the cell such as DNA/RNA, ion-channel proteins, microtubular proteins and/or cytosolic proteins, that apart from their chemical signalling may communicate through their vibrational character [16] [26] [41].

3) Cyclic polarized EM fields that can penetrate life tissue promote coherent vibration of various cell components [31] [42].

4) Extended fields of coherent molecules were assumed to be formed by a process called Bose-Einstein condensation that was proposed to occur also at life temperatures [26] [31].

5) EM photon, phonon and electron wave packages can be converted to mixed forms (quasi-particles) such as polarons (phonon/electron modalities, also called solitons), polaritons (photon/electron wave package [38].

6) Solitons in the cells may be able to constitute local quantum fields that both can be involved in intracellular morphogenetic ordering, as well as intercellular communication. For example, solitons have suggested to be instrumental in 3-D folding of proteins and DNA [10] [11] [39].

We found that the different measured typical frequencies in protein systems agree with calculated algorithmic soliton frequencies: oscillations of calcium-binding proteins in the cerebellar cortex of mice [43] and with spectral resonances of signaling proteins alpha-amino acids in the ERK-MAP Pathway [44]. The vibrational features detected by us also are fully in line with the frequencies related to tubulin protein self-assembly [41] [45] [46] [47], the microwave radiation frequencies of green fluorescent proteins [48], the frequencies present during synthesis and protein folding [49] and even to the resonances of aberrant methylation sequences in DNA [50]. 
Making use of the obtained algorithm of soliton frequencies, the discrete EM frequency bands and corresponding geometries, insight may be given in the patterns of folded proteins by down scaling the soliton patterns of acoustic waves to a (sub) micron level at a Terahertz frequency scale, as once made visible by Chladni (1787) [37] and Ritz (1909) [36], see Figure 3. According to the GM scale model of Meijer and Geesink, octave hierarchy of frequency patterns exists, and the revealed geometries are embedded in each other, as made visible by the nested fractal torus model. By selecting the right soliton basic frequencies alignment of patterns, in principle the folding of "proteins" can be made visible. In addition, rotations of structures have been measured [49]. We have recently defined the mathematical basis for the GM-scale [11] and found that the discrete distribution pattern of electromagnetic frequencies perfectly fit literature data for EPR-entanglement promoting EM fields as well as known mass/energies of all of the elementary particles of the Standard model [51] [52].

\section{Potential Roles of Electromagnetic Force Fields in Biological Evolution and First Life}

The highly reproducible and mathematically defined EMF (electromagnetic field) frequency bands (eigenvalues) that result in life sustainment of living cells, are envisioned as a permanently operating wave pattern phenomenon, [10] [11], and turned out to be strikingly similar to discrete EM (electromagnetic) radiation frequencies of typical clay minerals that have been shown to display semi-conductive properties and act as quantum wave replicators [10] [38]. Such mineral substances may have been instrumental in the creation of first life in relation to the steering influences on overall cell morphology, structure of macromolecules such as proteins, as well as directed synthesis of poly-nucleotides [53].

Sond-induced geometric patterns on thin vibrating plates: Chladni and Jenny
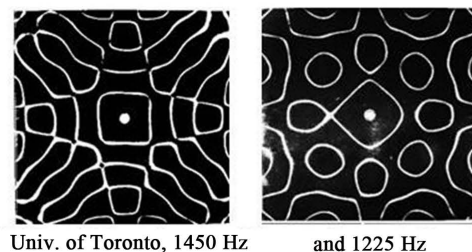

and $1225 \mathrm{~Hz}$
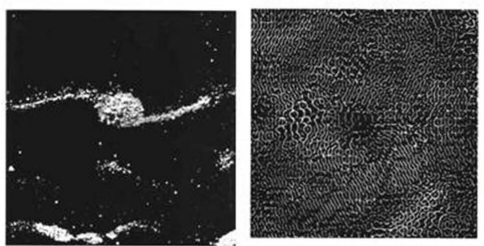

Jenny, Rotational effect, $12.47 \mathrm{~Hz}$ and $17.600 \mathrm{~Hz}$

Three different Chladni patterns demonstrated in:

An amazing Resonance Experiment: https://www.youtube.com/watch?v=wvJAgrUBF4w

Figure 3. Analogy of phonon-guided protein folding and acoustically induced complex geometric patterns. The latter are evoked on particle covered thin plates, as earlier separately demonstrated by Chladny and Jenny (see also reference: "an amazing resonance experiment" found on internet, see https://www.youtube.com/watch?v=wvJAgrUBF4w). 
Of note, this type of minerals, apart from being present in soil, is also abundantly suspended in the earth ionosphere (so called atmospheric dust) and have been suggested to provide a semi-conductive medium that produces selective EM wave patterns following excitation by external energy sources. It is of interest that such silicates have been reported to be candidates for the facilitation of oligo-nucleotide synthesis in the creation of first life in biological evolution [54]. Elements in the universe have seemingly assembled themselves in such a way that the organization of matter resulted into the ability to acquire sufficient life-sustaining information from the environment. Over time, highly complex neg-entropic structures arose that could collect, store, retrieve and communicate essential information to maintain stability and survivability. Such pattern recognition ability may have been directed by combinations of EM radiation frequencies through inducing morphic resonance with coherent vibrational elements (structured water, proteins, oligonucleotides) of proto-cells.

According to Wolynes [5] the integral folding process includes random mutations, potential misfoldings/unfoldings, recombinations and selection by successful competition with less optimal protein species, in which the protein finally obtains sufficient stability in subsequent generations of cells. As such the proteins were seen by the author as non-linear elements in cellular networks that arise from a sort of information spaces that unfortunately, were not further defined. One could also question the supposed random character of this self-organizing process. In other words, how can selection of non-functional precursors of the particular protein be envisioned? And by what physical mechanism is a specific function assigned to the particular protein? As argued by Grandpierre [55] life functions of proteins cannot arise by chance, they can only be assigned by their host cell, but such a cell cannot arise without these functions being already assigned. We propose therefore that a primordial biological principle (register of rules) was operating, that acts as a "recipe for life" [56]. This type of a-priory information must have preceded the development of first life and all known force fields that were present from the birth of the universe should be taken into account (see later). Also quantum processes have probably played an essential role in facilitating the various steps that gave rise to first life and initiation of the first replicating cells [57], see Figure 4.

A part of the EM frequencies at stake, were shown by others to be involved in phonon and soliton- (and thus sound-like) mediated steering of cellular functions [32] [33] [34] [58]. It was inferred that the discrete frequency bands (also called eigenfrequency values), as identified in the meta-analysis of the life studies, likely reflect a cellular regulation and communication system that may have an evolutionary origin, realizing that due to the composition of our planet, EM radiation is a basic property of the planetary environment. Life on earth was formed during billions of years, exposed to, and shaped by the original physical forces such as gravitation, cosmic irradiation, atmospheric electric fields and the terrestrial magnetism. The Schumann resonances at $7.8 \mathrm{~Hz}$ and a series of other 
The miracle of the first cell: the potential role of quantum processes

- Superposition and entanglement of wave information

- Central information search in an environmental pool

- Parallel processing and selection of non-living states

- Goal directed backward causation due to time symmetry

- EM radiation in clay minerals ordering quantum replication
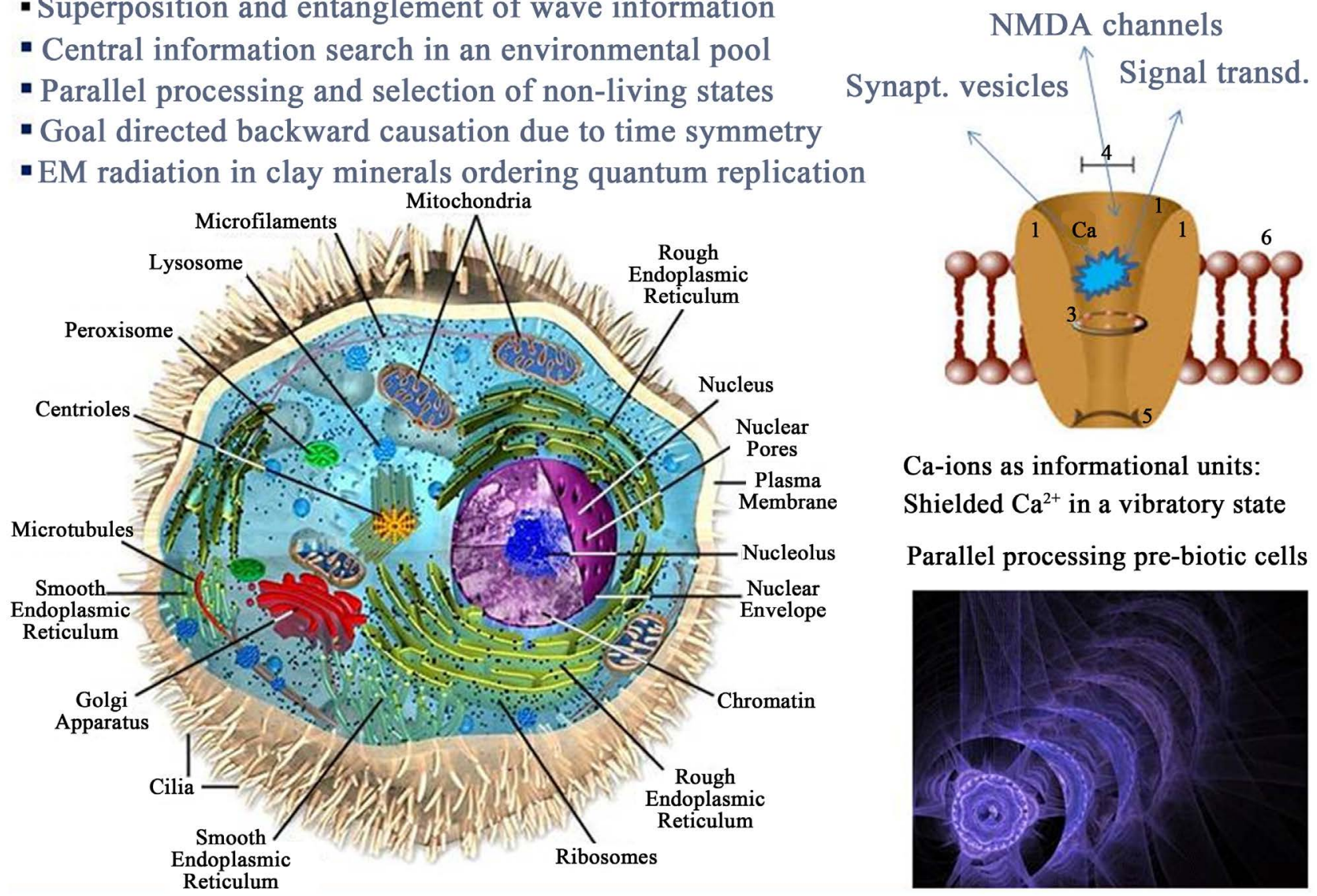

Ca-ions as informational units: Shielded $\mathrm{Ca}^{2+}$ in a vibratory state Parallel processing pre-biotic cells

Figure 4. Potential role for quantum processes in biological evolution (listed left above) and the initiation of first life, showing various essential processes. Inset Right above depicts a $\mathrm{Ca}^{2+}$ channel protein with $\mathrm{Ca}^{2+}$-ion in a decoherence protected vibratory quantum information state. Inset right below indicate the process of parallel processing of pre-biotic life information as enabled through environmental search of various quantum states.

related frequencies, are an example of oscillation that are potentially important for life. We conclude that the existing organisms are created to function in harmony with the above-mentioned fields and forces which existed when life was born 3 billion years ago.

This collective picture indicated that a distinct biological/physical/mathematical principle may operate in both non-animated and animated systems and that these discrete EM frequencies may have bridged information processing required for the creation of first life at pre-biotic conditions [1] [57] [59] [60].

\section{Soliton-Mediated Mechanisms of Protein Folding}

The relevant mechanism of electromagnetic wave interaction has been suggested to be spontaneous breakdown of symmetry in the biological, well ordered structures. Such interaction occurs with the dipole moments of the molecules in the cell microtubules [15] [45] [46] [61] [62] [63]. The biophysical aspects of solitons were adequately reviewed by Foletti and Brizhik [64] showing that in cells 
the quasi-particle is produced from an electron and a self-induced local lattice deformation of the molecular chain, propagating at constant velocity without energy dissipation, and emits radiative harmonic EM waves that facilitate the creation of coherent states of interfacial water molecules. Interestingly, the energy released under hydrolysis of an ATP molecule, the oscillation energy of the $\mathrm{C}=\mathrm{O}$ of the peptide groups and the different bending modes of interfacial water molecules fit precisely with the calculated soliton frequencies of the acoustic wave-function, respectively: $0.415 \mathrm{eV}, 0.2073 \mathrm{eV}$ and $1660-1693 \mathrm{~cm}^{-1}$. Also the $1.1 \mathrm{THz}$, mentioned by Brizhik, [14], fits exactly with the calculated algorithm of solitons related to a stabilizing coherent frequency.

Solitons are seen as localized, non-dispersive excitations, which exist in many nonlinear systems [65] [66] and have been proposed to act as an organizing principle in the process of protein folding [67], including membrane proteins [68]. They are very stable, and therefore can propagate without much energy loss or dispersion to much larger distances than wave-packets of linear waves. In proteins, the dipole-dipole interaction between neighboring amide-I (the $\mathrm{C}=\mathrm{O}$ double bond) quantum modes of vibration gives rise to linear collective modes known as excitons. The dipole-dipole interaction is influenced, however, by lattice vibrations, so the excitons and topological solitons [68] interact with acoustical phonons in the protein and/or provided by quasi-particles. The energy that is carried by the soliton is transferred into the conformation field, and this allows it to overcome energy barrier and reach the desired ground state. It might be that solitons are generated in locations of preferable alpha-amino-acids sequences, while their propagation might be blocked on different sequences. Therefore, the sequence may not only dictate the final conformation, but also the dynamics of the conformational transitions (Figure 5).

Among these, collective excitations driven by metabolic activity were hypothesized by $\mathrm{H}$. Fröhlich to account for the huge speed of enzyme reactions and for the fast encounters of the cognate partners of biochemical reactions. In fact, according to this hypothesis, collective oscillations of biomolecules, by bringing about giant oscillating dipole moments, would result in resonant (thus selective) electrodynamic forces acting at a long distance. The particular model assumes that the activation mechanism of collective oscillations can be seen as a Bose-like condensation of the normal vibrational modes of a bio-molecule. In this model a bio-molecule is considered as an open system through which energy flows under the simultaneous action of an external supply and of dissipation due to radiative, dielectric, and viscous energy losses. Such Bose-like condensation, in the lowest vibrational mode, is predicted to occur when the energy input rate exceeds some threshold value.

Recently, Nardecchia [28] highlighted the process of far distance oscillation phenomena in proteins. The authors stipulate that the condensation phenomenon originally proposed by Fröhlich as a quantum process, should be discussed, because the supposed quantum conditions can probably be hardly maintained for biomolecules. In fact, the frequency of collective oscillations is expected in 


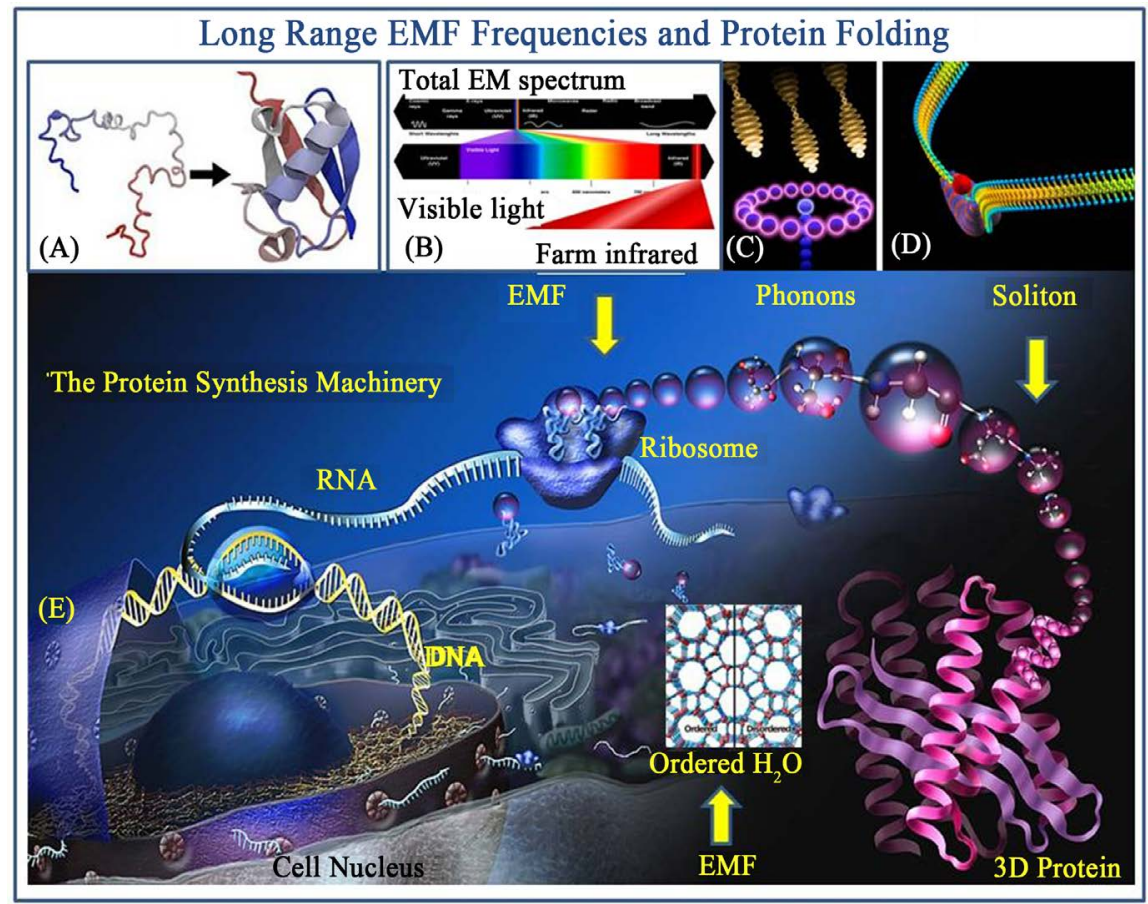

Figure 5. Soliton/phonon guided protein folding, depicting protein synthesis from DNA/RNA (left) to linear amino-acid chain (middle) and final folding to 3-D conformation (right). Inset in the middle shows: EMF-mediated structuring of water in hexagonal geometry, and on top, (A): Protein folding from linear AA chain; (B): electromagnetic field spectrum with visual and far-infrared parts; $(\mathrm{C})$ : phonon induced coherent vibration domain (BE-condensation) and (D): soliton/polaron travelling along a protein backbone.

the sub $\mathrm{THz}$ domain, around $10^{11} \mathrm{~Hz}$, so that at room temperature $\mathrm{kT} / \mathrm{hv}>>1$, which may rule out a quantum description. An additional, more classical approach on the macromolecular vibratory communication should be considered even though experimental evidence of the existence of collective modes of vibration of biomolecules has been provided at thermal equilibrium by means of Raman spectroscopy already years ago by Lundholm [26]. Yet, this was measured in a quite artificial setting: crystallized proteins. Accordingly, Nardecchia worked out a classical version of the original Fröhlich model, finding that, remarkably, also in a classical context a Fröhlich-like phonon condensation phenomenon is possible. This was illustrated by the authors, for an idealized macromolecule, displaying the deviation from energy equipartition among the normal vibrational modes. Secondly including collective modes of resonances of biomolecules at infrared/light induced electronic transitions may balance $\mathrm{kT}$ [11].

Though still representing a biomolecule in a very idealized way, this model predicts a classical condensation phenomenon, that seems worth to be experimentally investigated. Of note, the same group earlier demonstrated long range resonant communication of biomolecules, by measuring their diffusion behavior, indicating an elegant experimental method to probe the activation of resonant electrodynamic interactions among biomolecules [28]. 


\section{The Essential Role of Cell Water in the Protein Folding Processes}

Cellular plasma water is generally supposed to act as a transfer medium for external electromagnetic waves to biomolecules for adequate introductions in this field see [69] [70] [71] (Figure 6). The cellular plasma was proposed to exhibit a highly arranged 3-D geometric structure as a liquid crystal that exhibits surface interactions with macromolecular structures. The absorption spectrum between $1 \mathrm{THz}$ and $10 \mathrm{THz}$ of solvated biomolecules is sensitive to changes in such fast fluctuations of the water network. There is a long range influence on the hydration bond dynamics of the water around binding sites of proteins, and water is shown to assist molecular recognition processes [72]-[81]. "Biological water" supports itself by coherent dipolar excitations and terahertz/femtosecond infrared interactions and these dynamics extends well beyond the first hydration shell of water molecules. The latter publication makes clear that water molecules in

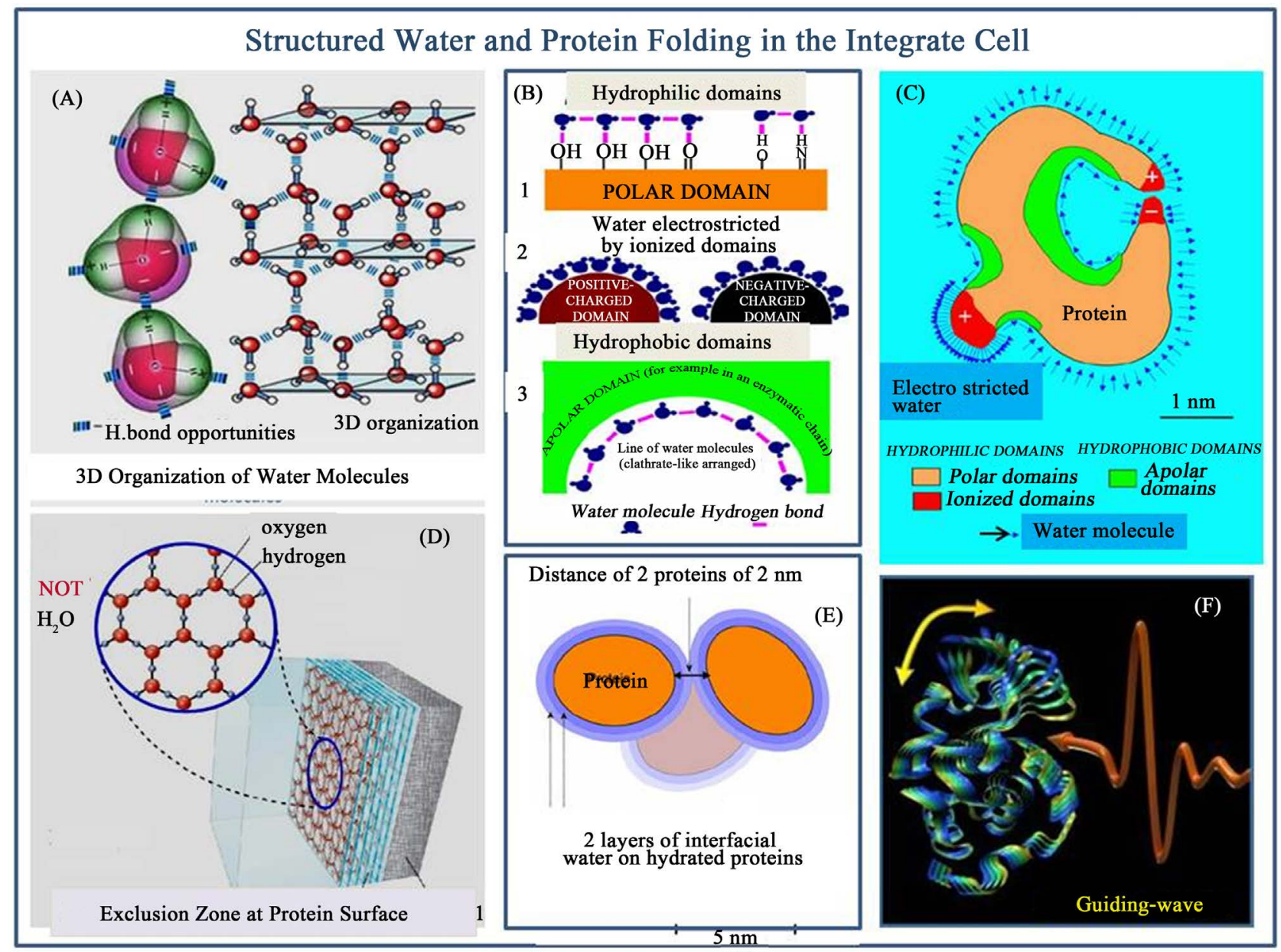

Figure 6. Various aspects of electromagnetically structured water complexes and protein folding. (A): Molecular conformation and 3-D hexagonal organization of water molecules; (B): Dynamical hydration layers in hydrophilic and hydrophobic portions of a protein; (C): The influence of protein hydration in polar and non-polar domains of the protein chain affects folding; (D): Formation of water exclusion zone at the surface of macromolecules (E): The crowded cytoplasm space with adjacent proteins covered with layers of water molecules and hydrated ions (F): EM field guided protein folding (after Mentré, 2012, and Brown, 2016). 
the interfacial setting with their dipole character, in combination with cytoplasm inorganic ions, interact with charged groups on the protein backbone, by which surface charges become masked and relatively hydrophobic domains are created that can lead to deformations (see Figure 2(C)) and creation of local pockets or cavities, that even may exclude acqueous solvent molecules. This adds up to the natural influence of both hydrophilic and hydrophobic portions of the particular protein chain that are involved in the initial folding process as well as protein stability [82].

Water molecules have typical resonances at Hertz frequencies, and a typical frequency can be expressed in 3-prime-limit tuning. A calculated typical frequency of a water molecule, with a molecular weight $\mathrm{M}=18 \mathrm{~g} \cdot \mathrm{mol}^{-1}$, is $54 \mathrm{~Hz}\left(2^{1}\right.$, $3^{3}$ ) according to Henry [49]. This typical frequency of a water molecule, in the centre of our GM-scale, can be derived by using the mass-energy equivalence coupled to the Planck-Einstein relationship: $\mathrm{M} \cdot \mathrm{c}^{2}=\mathrm{h} \cdot \mathrm{f} \Rightarrow \mathrm{f}(\mathrm{Hz})=2.981 \times \mathrm{M}$ $\left(\mathrm{g} \cdot \mathrm{mol}^{-1}\right)(\mathrm{M}$ is molecular weight of water molecule, $\mathrm{c}=299,792,458 \mathrm{~m} / \mathrm{s}, \mathrm{h}=$ $6.62606959 \times 10^{-34} \mathrm{~J} \cdot \mathrm{s}, \mathrm{f}$ is the frequency of a water molecule). In two papers of Sbitnev [79] [80], a relation between Bohmian pilot wave guiding to toroidal vortex topologies and zero-point energy was discussed in the framework of cell water hydrodynamics.

In this respect, some innovative papers with designed and properly controlled experiments, suggested that electromagnetic fields may reflect macromolecular features of enzymes like DNA-polymerase in water, and that this provides structure specific information storage in aqueous solutions [82] [83] [84]. The electromagnetic radiative properties of DNA and enzyme macromolecules likely depend on the characterization of their electric dipoles. The molecular structure of DNA base pairs and enzyme amino acids, as well as many other bio-molecules, presents aromatic rings containing conjugate planar systems with $p$ electrons delocalized across the structure. It has been argued that London force dipoles in such intra-protein hydrophobic pockets could couple together and oscillate coherently, thus generating a radiative field. Montagnier recently described the fundamental role of water dipole dynamics bridging the interaction between DNA and enzymatic proteins (see Figure 7) so as to allow polymerase chain reaction (PCR) processes to occur [84]. They describe the dynamic origin of the high efficiency and precise targeting of Taq activity in PCR.

The spatiotemporal distribution of interaction couplings, frequencies, amplitudes, and phase modulations comprise a pattern of fields which constitutes the electromagnetic image of DNA in the surrounding water, which is how the polymerase enzyme actually recognizes in the DNA water environment. The DNA and the enzyme "see" each other's EM images by exchanging quanta of the radiative dipole waves induced by their presence in the water molecular matrix, which thus acts effectively as a bridge between the two, until they are sufficiently close for water exclusion and direct binding to occur (Figure 7). This standpoint is consistent with previous experimental work that has shown that some DNAs in water dilutions have electromagnetic waves of low frequency (from hundreds 
to $3 \mathrm{kHz}$ ) which also could be triggered, as in a resonance phenomenon, by an outside excitation, such as Schumann waves. It is a great interest that macromolecular and drug molecule vibrations can be imprinted and informational encoded in stable ordered coherent water domains by photon EM irradiation, as experimentally demonstrated in various studies with a spectrum of different therapeutic agents (reviewed by Lawrence [85], for potential nontoxic treatment.

We propose in this respect that the cytoplasmic macromolecular aqueous solution is ordered via the the discrete wave frequencies that have been described by us [11], and that this exhibits a fractal self-similar pattern around the central 12-tone octave frequency bands. It is of interest that a fractal-like self-similar structure induces the manifestation of coherent dynamics active at a microscopic level. These (fractal-like) self-similarity properties of the EMS in turn likely induce self-similar organization in the irradiated water. This first step can be followed by a second step consisting of transformation of the signal into an analog form and sending its electric vector to a solenoid which than will generate a magnetic field in the surrounding water. The action of reciprocal "seeing" is described by the exchange of the dipole wave quanta of the collective dynamics induced by DNA and enzyme in the surrounding water dipole field, as derived above. The general process is described by the analysis of two interaction "vertices": the interaction vertex DNA-\{water dipole wave\}, on the one hand, and the interaction vertex \{water dipole wave $\}-T a q$, on the other. What this study provides is the dynamical description of the long-range EM correlation modes orchestrating the PCR process, thus accounting for its remarkable efficiency, space-time ordering, and diverse time scales that would be unattainable by otherwise fully stochastic molecular activities.

Of note, divalent cations such as $\mathrm{Mg}^{2+}$ and $\mathrm{Ca}^{2+}$, in particular, may enhance or maintain or critically deform the long-range dipole wave fields in water solution. This is supported by recent experimental work that shows that ions can influence up to 100.000 water molecules, which extend roughly $5 \mathrm{~nm}$ from the ion radially. This would add another modality of form information regulation. Mc Dermott [86] reported their discovery of a chiral water superstructure surrounding DNA under ambient conditions. They confirmed the existence of a DNA minor groove spine of hydration at room temperature and further showed that the chiral structure of biomolecules can be imprinted on the surrounding solvation structure. The observation reported in of the robustness of the DNA's chiral spine of hydration, and the fact that "a change in the hydration state can lead to dramatic changes to the DNA structure" also indicate that such a water superstructure actually constitutes a detailed mold or "electromagnetic image".

All this highlights the crucial role of water as a connecting modality in the EM vibrational steering of 3-D macromolecules and also in the realization of holographic memory of the cell and entangled information transfer in the universe as a whole [76]. In the latter study it was emphasized that in the interfacial water layer of proteins both permittivity and viscosity of water is largely different from bulk water. Previously it was reported that long-range radiant energy excludes 
colloidal and molecular solutes from hydrophilic zones of proteins in a wavelength dependent manner [75].

It can thus be conceived that multiple bio-solitons of different discrete energies, facilitated by their quasi-particle phonon features, travel along the primary protein backbone (Figure 5), thereby providing essential long or short range information, that leads to the final conformational shape of the molecule (see for folding mechanisms later on). The various solitons could arise from external EM fields, preferably in the polarized form in relation to a more optimal tissue penetration or being formed internally and organized in coherent domains or collective modes in the cell. The question should be raised here how the particular series of travelling solitons translate the fine-tuning forces that finally shape the protein in its functional 3-D configuration. If special combination of solitonic wave frequencies would play a role, some sort of premordial or evolutionary harmonic information could be responsible (see later). Alternatively, the discrete EM energies may be derived from an addressable memory space for geometric forms of life macromolecules that have been build up in biological evolution.

The latter aspect will be treated in the following from the point of view of holographic memory space models. It should be mentioned here that apart from the coherence promoting soliton waves, also soliton frequency bands were identified by us that produce detrimental and de-coherent influences. Thus misfolding or destabilization of proteins could be related to such de-coherent EM radiation fields as have been suggested for cancer [87], and even in cataract and Alzheimer disease [88]. The general properties of solitons are solitary waves (waves localized in space) with the following properties: 1) they preserve their shape and velocities; 2) they are extremely stable to perturbations (in particular collisions with small amplitude linear waves); 3) they are even stable with respect to collisions with other solitons. In such collusion they pass through each other and recover their speed and shape after interaction. It should be noted here that phonon density on electrons can largely determine the harmonic versus an-harmonic features of such polarons and thereby their diffusebility in life tissues and macromolecules [89].

\section{Protein Folding in the Intact Cell: Do We Need More Integral and Holistic Approaches?}

It has been firmly established that in vitro protein folding models do not correctly predict in vivo folding in the whole cell context (references in [30]). In fact the many factors depicted in Table 1 , to some extent may contribute to the final 3-D functional structure, depending on the particular protein at stake. This addresses the general item how nature makes use of neg-entropic information related to the wide variety of life systems, including the morphology of crucial macromolecules that were created during biological evolution. It stands to reason that somehow this information is registered and re-used to prevent redundancy [59] [90] [91]. This would require a addressable quantum register or a 
dynamically build memory space that allows a versatile searching modality and potential parallel processing of information of very different structures in the various contexts that nature offers (see Figure 4). For example such a search procedure could integrate genomic and proteomic knowledge on the basis of wave interference patterns that can be stored through holographic memory processes probably related to a background quantum field. This not only introduces a huge memory space, but also implies that a holistic framework is created, in which any small fraction of the hologram reflects the total information pattern. Interestingly, such a mechanism was earlier used for the holographic storage of information in the human brain [92] [93], and is currently also used in physics and cosmology for the geometric description of radiating black holes, with the known assumption that quantum information cannot be lost. Such processes could play a role at any fractal scale if the universe, including the cells that constitute life organisms and their macromolecular components. As Schrödinger stated for living matter in general, the attempt to explain the high efficiency in terms of ordering generated by "statistical mechanisms" would be "the classical physicist's expectation that far from being trivial, is wrong", and "it needs no poetical imagination but only clear and sober scientific reflection to recognize that we are here obviously faced with events whose regular and lawful unfolding is guided by a mechanism entirely different from the probability mechanism of physics".

\section{Steering by Holographic Memory Hypersphere of the Individual Cell}

Each individual cell in our body is not a "stand alone" information processing unit: it acts as a part of our integral organism with recurrent information exchange or permanent feedback coupling with the entire organism. Such an integral context attracts much attention in cosmology, physics and also in life studies and is often approached with, what is called, the holographic principle [94] [95]. The holographic principle states that everything that is embedded in a space region can be described by bits of information at its border. In view of this basic definition there is no reason whatsoever not to apply it to complex life cells that obviously can be seen as bundles of integrated neg-entropic information. Holographic principles may clearly provide the information channel required for any biological system, in order to keep track of all essential information at once, and creating a dedicated memory space for sustaining life [96].

In this respect it was postulated earlier that the intrinsic information that defines the living condition of organisms may be holographically stored on a virtual screen associated with the particular life system [97]. The idea of a holographic organization of memory even in single cells was earlier proposed by Anjamroos in relation to preventing reprogramming of differentiated cells and activation of immune responses [98] [99]. This non-genetic memorization was claimed to be generated in the cell both endogenously and exogenously at several 
levels of the cell such as atoms, molecules including proteins, cellular water, microtubules and electromagnetic fields. The collective information may be projected in a holographic manner in a memory space from which every part of the cell is informed about the integrate hologram information of the particular tissue type (Figure 7). This information is considered to be non-local, a feature that in quantum physics is related to the phenomenon of entanglement. We have recently shown that discrete EM frequency pattern precisely fit earlier EPR experimentation in which such EM radiation promotes states of quantum entanglement [51].

Hales pointed out that the plasma membrane of the cell contains a high density of ion-channels that, in a collective action, can produce a major migration of charged ions, a phenomenon that may induce local magnetic forces [100].

These take the form of toroidal organized force vectors that even may extend the cellular membrane surface. This process could be enhanced due to GM-like coherent vibrations that, as mentioned above, have been shown to increase the degree of quantum entanglement [51]. Such a process can especially be anticipated in the quantum de-coherence protected channel interior [101]. This kind of external magnetic domain could provide the opportunity for wave interference with EM fields in the environment, and could in a holographic manner

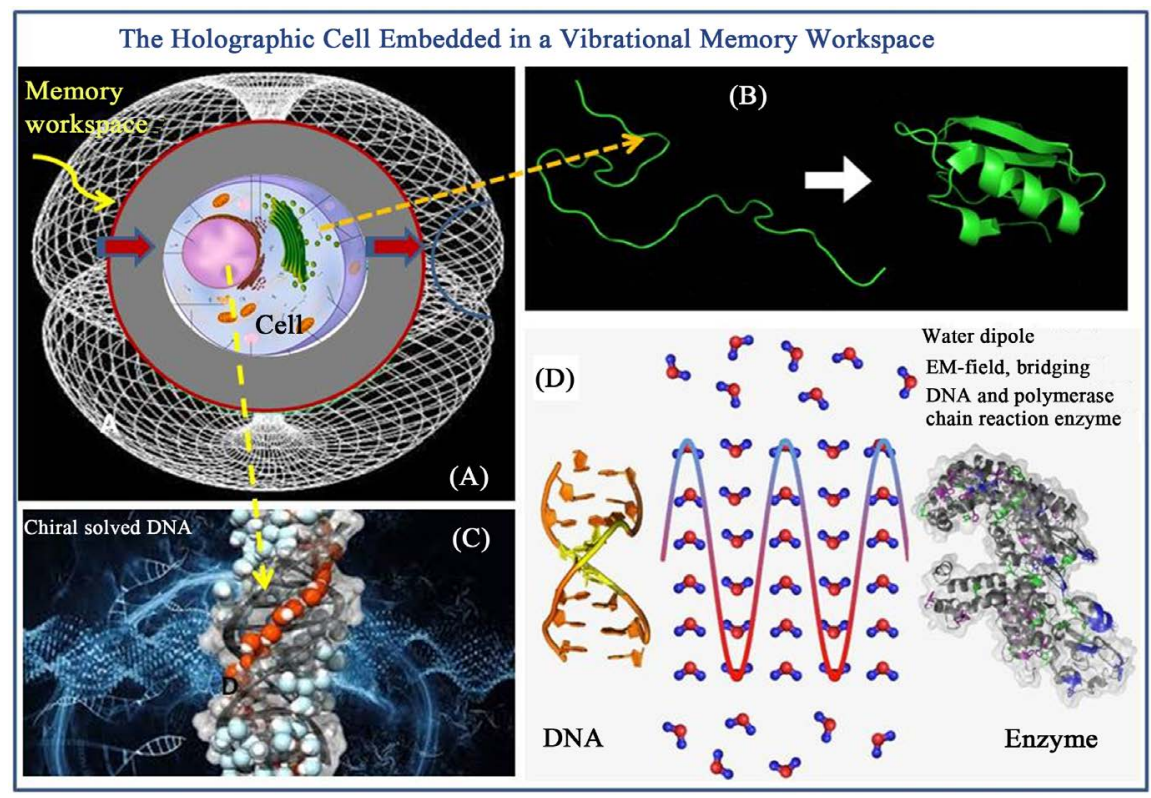

Figure 7. Toroidal hypersphere mediated morphogenesis of the integral cell. (A): The nested torus structure with an outer surface of the torus (red circle and arrows) represents the event horizon memory space surrounding the cell; (B): Depicts the 3-D folding of life macromolecules as it occurs following primary synthesis in the cell; (C): Shows the fractal attractor structure of $\mathrm{H}_{2} \mathrm{O}$ around DNA, steered by quantum information of the toroidal hypersphere memory workspace; (D): Shows the dipole radiative EM field of arranged water molecules that bridges the DNA molecule and PCR enzyme as essential for realization of the PCR activity. The coherency of cytoplasmic domains may be steered by externally applied EM fields in a resonance state with the various cellular vibratory elements (modified from Montagnier, 2017). 
build up a dedicated memory space for each cell, containing stored and updated life-information for cell survival. Such a cell bound memory space could also be responsible for long-distance, non-local, communication between different cells, as reviewed by Cifra [102] and experimentally shown by Fahradi [103].

A bio-photon type of information transfer was suggested in the latter paper and Persinger and Dotta even demonstrated that cell-specific wave information can be spatially stored for quite some time, although the physical mechanism of this space-time phenomenon remained unknown [104].

Several studies have implied that in pre-biotic evolution various force fields may have been instrumental in the creation of fist life [1] [47] [59] [60]. If a cellular holographic memory space would be involved it may imply that discrete EM wave-frequencies from the photon sea of zero-point energy can directly influence cell function by wave interference and holographic storage and thereby can also guide the 3-dimensional folding of life macromolecules into functional units of the information networks of the cell. In relation to this it was earlier stipulated by Gough and Shacklett that a knowledge domain beyond the Planck scale could provide characteristics of the well known implicate order of David Bohm [105].

There is recent evidence that such a domain outside known space-time could be constituted by a spin-liquid network [106], later pictured as a geometry of relations coined as the amplituhedron [107] [108]. This underlying domain contains information expressed in mathematical and geometric wave relations and can be inferred from a mathematical operation through transformation into twistor space. By some this is called a frequency domain that could function as a source of Bohmian pilot waves [12]. Such a pilot wave-frequency domain could contain a primordial recipe for the representation of neg-entropic information that may have led to the constitution of first life and also as proper information space for the maintenance of present life (see Figure 8). It was recently shown that a photon that is removed from our physical world can still become entangled with another photon with similar features in the present world, in spite of the fact that they never co-existed [108].

This is strongly indicative for an information domain beyond our local 3D-space time in which information of the destroyed photon is somehow stored. Thus material particles in general should be seen as excitations of an underlying non-material matrix that behave as vortices or perhaps as tori in a 4-D setting, also producing quasi-particles such as polarons/solitons. A deeper (geometric) information domain was also implied in "Our Mathematical Universe" by Tegmark [109] (discussed by Butterfield [110]) as well as for the life world by Azevedo and Filho [111] and Fleming [112], the latter postulating a fractal circular wave guide, involved in intra- and inter-cellular communication.

This cellular memory aspect was coupled to the assumption that cells are embedded in a 4-D toroidal structured topology (as proposed by Tozzi [113], Haramein [114]), that interacts with resonant sensitive energy structures in the various cell compartments, including their proteins (Figure 8). This idea of 


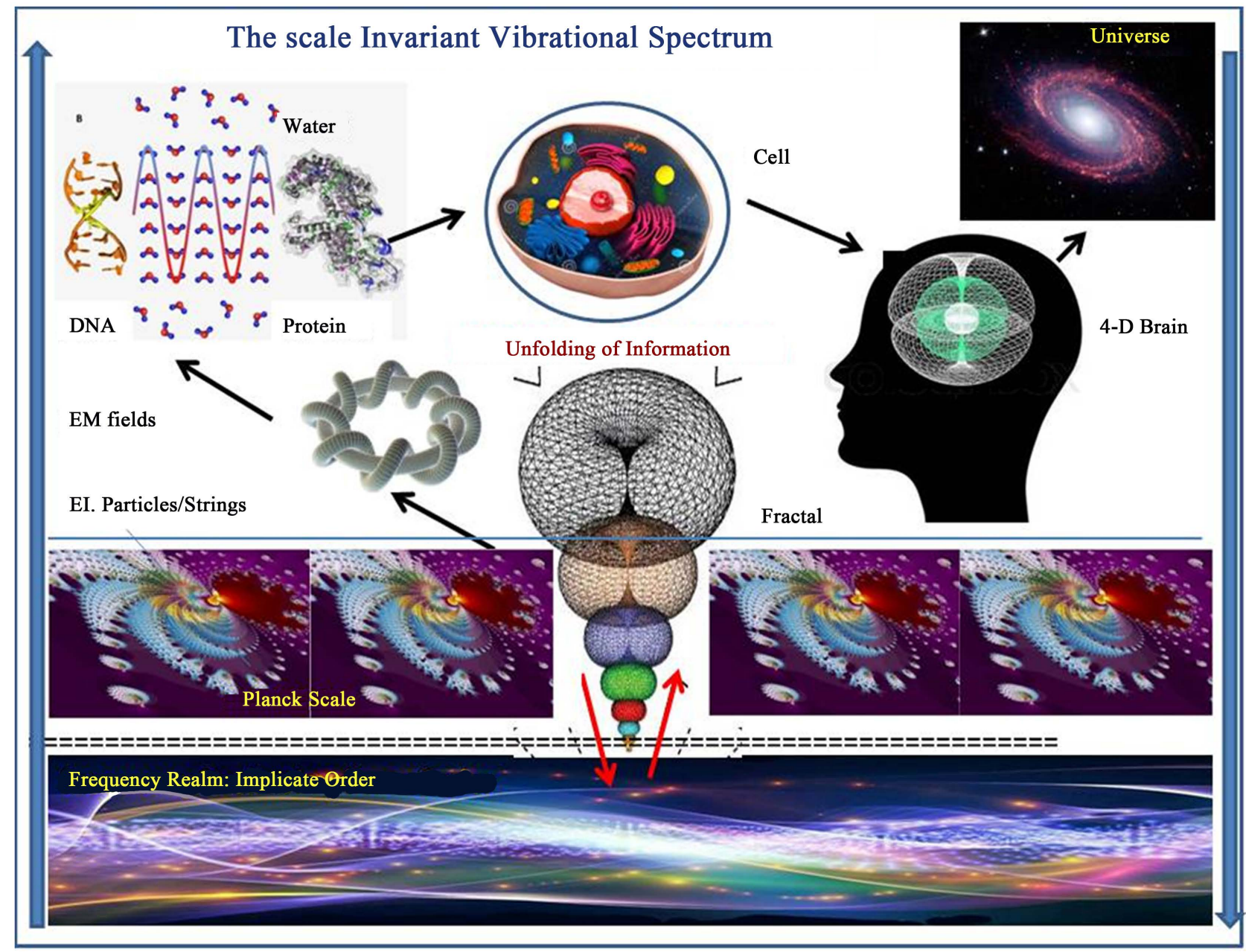

Figure 8. Morphogenesis of reality seen as a fractal process of information processing (collection, compression and subsequent unfolding), either (bottom up) from an implicate order frequency domain (below) up to the level of the Planck scale, and at higher levels producing elementary particles, atoms, life molecules for the build-up of whole structure of the cells and organs such as the brain. All elements are equipped with a toroidal memory workspace (consciousness) that is scale invariant (see Meijer and Gessink, 2017). In this manner it finally results in to black hole/white hole structure that (top-down) transmits information for the fabric of reality, thereby constituting a circular (rebound) type of information universe (see large arrows at the sides for bottom up and top-down information processing).

extended spacetime was earlier agreed upon by many others in physics, and implies that our reality and thus life processes are considered to be embedded in a 4th spatial dimension that we cannot observe but through symmetry breaking can infer from shadow energies in our world [10] [114]. In this respect, the geometry of the nested torus that also accommodates 4-D aspects, is envisioned as a scale invariant basic unit (operator) of spacetime, with unique collecting, coupling and integrating properties for a wide variety of wave information (Figure 8). These include photons, phonons and quasi-particle/waveforms such as polarons/solitons and polaritons, as recently discussed in our studies on phononand soliton-guided biology [48] [115].

Bio-photons have been widely proposed as information carriers in brain [63] [115] [116] [117], but also in other tissues [118] [119]. Recently it has been 
shown that incubation of brain slices with glutamate (a major neurotransmitter in the brain) results in the release of red-shifted bio-photons [120] and in a recent PNAS paper that the degree of bio-photon induction in brain slices of various species is highly correlated with the relative intelligence of the species studied [121]. These studies confirm the idea that bio-photons are instrumental in ultra-fast cellular communication, in addition to the known action potential mediated messaging and may even be central in building up of consciousness. We infer that the communication between a holographic memory space of the cell with its components could be bio-photon mediated, and that coherent soliton/polaron activities, indicated above, can lead to guiding of protein folding against the background of a bio-photon equipped hologram of the entire functional structure of the cell.

The cell, within such a memory hypersphere, is conceived as a dedicated multi-cavity network that for instance can convert ZPE wave information as well as states of gravity, dark energy and earth-magnetism, in an attractor type of fractal energy fields. The hypothesized external, field-receptive memory domain, may store wave interference patterns as defined by the abovementioned holographic principles. This generalized principle also implies that every small part of the hologram contains all the information of the whole picture [101] [102], and thus a large number of holographic fractal planes or octaves [10] [122]. We have earlier applied this holograhic concept to the entire brain [104] and in view of the known fractal character of the neuronal system [96] [122]. We consequently assume this to occur also at the various brain scales (neuronal networks, individual neurons, membrane and cytoplasmic proteins and ions, as well as constituting atoms and elementary particles).

As treated above, we therefore have postulated a self-similar, fractal, type of holographic information processing to be expressed in a holonomic entangled matrix of the whole complex neural structure including the various cell types of the brain [10] [122]. We assumed that a toroidal geometry that facilitates self-referential and spiral energy trajectories, functions as a basic operator unit for information integration processing and transfer 122, as was previously proposed for the mathematical transition to twistor space [123]. As mentioned earlier, by Persinger and Dotta showed that patterns of photon emissions can be stored and retrieved after some days from the same defined space and that collections of cells in culture in different loci can share the same complex magnetic fields, suggesting a non-local geomagnetic space time connection, that was explained by involvement of optical holograms [104].

Although the step that we take here from the whole brain to individual cells seems huge, it should be realized that even single neurons have been suggested to be able to develop conscious states [93] [124] [125] [126]. Also in biological evolution the first primitive proto-cells required a dedicated and addressable memory space to store stimulus/reception/action type of information in a learning process to cope with problem solving in the survival in very hostile environments [57]. Since, even in pre-biotic times, all the known force fields were 
present and the becoming cells should gather essential ecological information from their direct surroundings and planetary atmosphere, such an integral memory space would be a prerequisite for survival. We argue that such a memory workspace associated with life cells should be field-receptive and apart from containing the integral cellular information for life, can collect and integrate external information from the mentioned force fields in which all life organisms are permanently embedded.

How would such a quantized memory unit, associated with the cell, communicate in a bi-directional manner (see Figure 8)? It is likely that wave resonance and wave interference represent viable mechanisms for this purpose, in which cellular energy stimulates coherent vibration states of intracellular components. This electrome background of the cell can in this manner undergo a holographic interaction with external and integrated wave information. Among others, the cellular architecture could be guided by discrete patterns of eigen-frequencies of solitonic waves directed at the cell and backwards. The latter process serves for updating a time-symmetric global memory space of the individual cell. Solitons or polarons are electrons "dressed" by phonons and are sensitive to cyclotron resonance [127]. It is well known that sound (noise) can largely facilitate effective transport of charged particles [93] [128].

If each cell would be surrounded by such a "memory space" that houses wave/particles with discrete wave frequencies, a toroidal coupling of phonons and electrons could provide soliton waves that may function as pilot waves in the Bohmian sense. If the cell contains coherently vibrating macromolecules, influenced by cyclotron resonance [127] and/or stochastic resonance soliton information from the proposed hypersphere memory workspace, could lead to guiding wave interference [129]. This can than lead to perturbation of the 3 -dimensional protein molecule structures, in this way being instrumental in the transfer of crucial life information that is stored in the 3-D conformation of proteins and poly-nucleotides. Using such a "memory mirroring", an individual cell could in this way build up a holographic endo-memory of the exo-world. Through the evident fractal character of the cell structure this could not only play a role on the molecular level but even at more deeper atomistic levels up to elementary particles and could even provide qualia-like information at the Planck scale [62] [96], see Figure 7.

At the atomic level, $\mathrm{Ca}^{2+}$ ions/waves could play a central messenger role since the ion is supposed to be an excellent information carrier [130], and is involved in a spectrum of regulatory processes in the cell. $\mathrm{Ca}^{2+}$-ions, noise protectively enclosed in $\mathrm{Ca}$-channels and $\mathrm{K}^{+}$-ions in $\mathrm{K}$-channels in the cell membranes, for instance, may produce quantum wave coherence states that endure long enough to transmit entangled information in and between cells [108]. $\mathrm{Ca}^{2+}$-ion trans-membrane gradients have also been shown to be very sensitive to cyclotron electromagnetic radiation [131]. In the dynamic, field sensitive, electrome state of the cell, directed protein folding to a functional 3-D conformation, would in this way be steered by an integral biophysical recipe. This 
recipe, in principle, encompasses all the necessary in-formation of the metabolic networks in which the particular protein functions in the intact cell, taking into account all the factors as listed in Table 1. A similar memory workspace as described here for an individual cell, was coined for the whole brain as "global workspace" [132], or "cloud" [93], or "event horizon", [97]. Electromagnetic field aspects of brain function are extensively reviewed [133] and with regard to consciousness [79] [80] [97] [134]. Sbitnev [79] [80], discussed the relation between Bohmian pilot wave guiding to toroidal vortex topology and zero-point energy in the framework of cell water hydrodynamics. The relation with photon emission of the human brain was highlighted [104] [120] [121] [135]. Interestingly, it follows that a "proto-conscious" state can in this manner be ascribed to each cell in the organism through continuous information matching with the supervening holographic field-receptive memory space, just like the permanent reading of genetic information that is intrinsically present in each cell in the organism. Holographic associative memory for pattern recognition and information completion in which a weighing function is included to ensure dominance profiles and focus, have been earlier described for neural networks [131].

The general concept is that this piloting process functions at any fractal scale of the cell-system and, more general, as an informative vortex principle that operates from the Planck scale to macro-scales of organisms [122]. Einstein and Minkowsky originally proposed a $3+1$ dimensional space-time ( 3 spatial and 1 symmetric time dimension), in which all time is laid out, while later in physics $4+1$ models were adopted, including an extra spatial dimension (Kaluza-Klein theory). More recently, Randall (see [136]), postulated the Randall-Sundrum model (also called 5-dimensional warped geometry theory) imagining that the real world is living in a higher-dimensional universe, described by warped geometry. In this generally accepted space-time concept, time has a bi-directional (symmetric) character that allows aspects of backward- or retro-causation. In the framework of protein folding, this implies that a primary structure of the protein can also anticipate a future (final functional) structure. This aspect obviously would drastically speed up the folding process and may explain some of the questions raised before in the present paper (see evolutionary aspects). The aspect of top-down causation (process feed-back) by information control is extensively discussed [137].

If in single cells such a memory steered mechanism would operate, a crucial question is how the integral information with regard to single cell constitution would be translated in a multi-cellular context. One possibility is that discrete EM wave frequencies promote states of entanglement that than could explain long distance correlation of information in any tissue or even organisms and thereby would obtain a distributed character. In a recent study such non-locality inducing effects of discrete EM fields were revealed in a meta-analysis of so called EPR experiments [51]. Alternatively the EM vibratory character of cells may produce electromagnetic magnetic fields in which the form information is shared in a long distance manner [97]. The fractal or holographic property of cell structure cell (many copies at different sites) can in principle imply that mo- 
lecular state information is also expressed in the fine-structure of cells like the components of membranes and organelles. As treated before, such processes could also be helped by stochastic resonance and/or phonon/soliton (acoustic) mediated flexibility of the cellular matrix [131] [134]. In this manner a convergent correlation matrix of information would become manifest that can guide the whole architecture of life systems.

Our hyperspace information concept of cell memory may have some correspondence with the known quantum holographic Hopfield-like biomolecular recognition models [138] [139].

This computational approach was applied in the framework of protein folding [30] [140], among others. Incidentally, the successful prediction of protein secondary structure was one of the first examples for applications of neural networks in which these significantly exceeded the performance of all other systems and even that of experts. This principle provides quantum computing an advantage in processing huge data sets. An example of the latter is the Hopfield model of content-addressable memory using the concept of attractor states. This content addressable memory allows to compute even incomplete input information, which forms the basis for memory and learning. In the Hopfield network each update of a neuron minimizes the network energy if possible. Memorized firing patterns are thus stored as stable attractors that become holographically coupled at fractal layers in a process of multi-level information processing. In this manner, also pattern recognition can be attained from very different biophysical and bio-informational aspects of proteins, so that the prediction of 3-D structure can be highly facilitated. The earlier mentioned work of Chen et al., for example, uses hybrid data of atomistic associative memory and data base memory in structural predictions [4].

Finally, we emphasize that the soliton mediated processes mentioned in our pa-per should be seen as a steering of the balance of folding and unfolding activities: Unfolded or disordered protein regions play a crucial role in flexibility and in-teraction of proteins and can largely bind inorganic ions such as $\mathrm{K}^{+}$and $\mathrm{Ca}^{2+}$ as well as organic compounds such as ATP. This, in turn, can lead to polarization of the associated and structured water layers. The resulting fractal "cell matrix" supports coherent vibrations and harmonic resonance and may induce bio-photon discharge that can produce holographic properties (reviewed in [141] [142]).

\section{Final Conclusion}

It is postulated that the problem of folding of bio-molecules, theoretically, requires an unrealistically large number of steps to find the correct state. Perspectives for solving this paradox are associated with an explicit incorporation of topology in the physical models of folding. This condition can perhaps be achieved through the construction of hybrid models based on the geometrical and physical approaches [1]. However, it is necessary to perform future experiments to identify the specific mechanisms underlying the actual forces between molecules (atoms). In particular, the actual problem is the possible existence of a collective 
action at a distance between the molecules. The presence of such long-range interaction can substantially change the folding model. Moreover, it could lead to a complete revision of our understanding not only of the folding of proteins but also of the work of molecular biological structures in the broadest sense.

In our opinion, therefore, it is crucial to approach the item of protein folding from a more "holistic" standpoint, in which all the interacting factors and intrinsic conditions of the intact cell should be taken into account (as depicted in Table 1). These include long-range force fields and local cell biological conditions such as structured water complexes, among many other factors. In particular, we show that abundant information is available in the literature on the role of soliton waves (phonon/electron quasi particles) that can be instrumental as guiding elements in the various steps in the folding process. This, by crucial energy transitions in their interaction with the semi-conducting protein lattices. Our recent finding of a set of discrete EM frequency bands that either promote or endanger life conditions (see Figure 9), could be a key in further studies

\section{Electromagnetic Resonance in Cells: the Electrome}

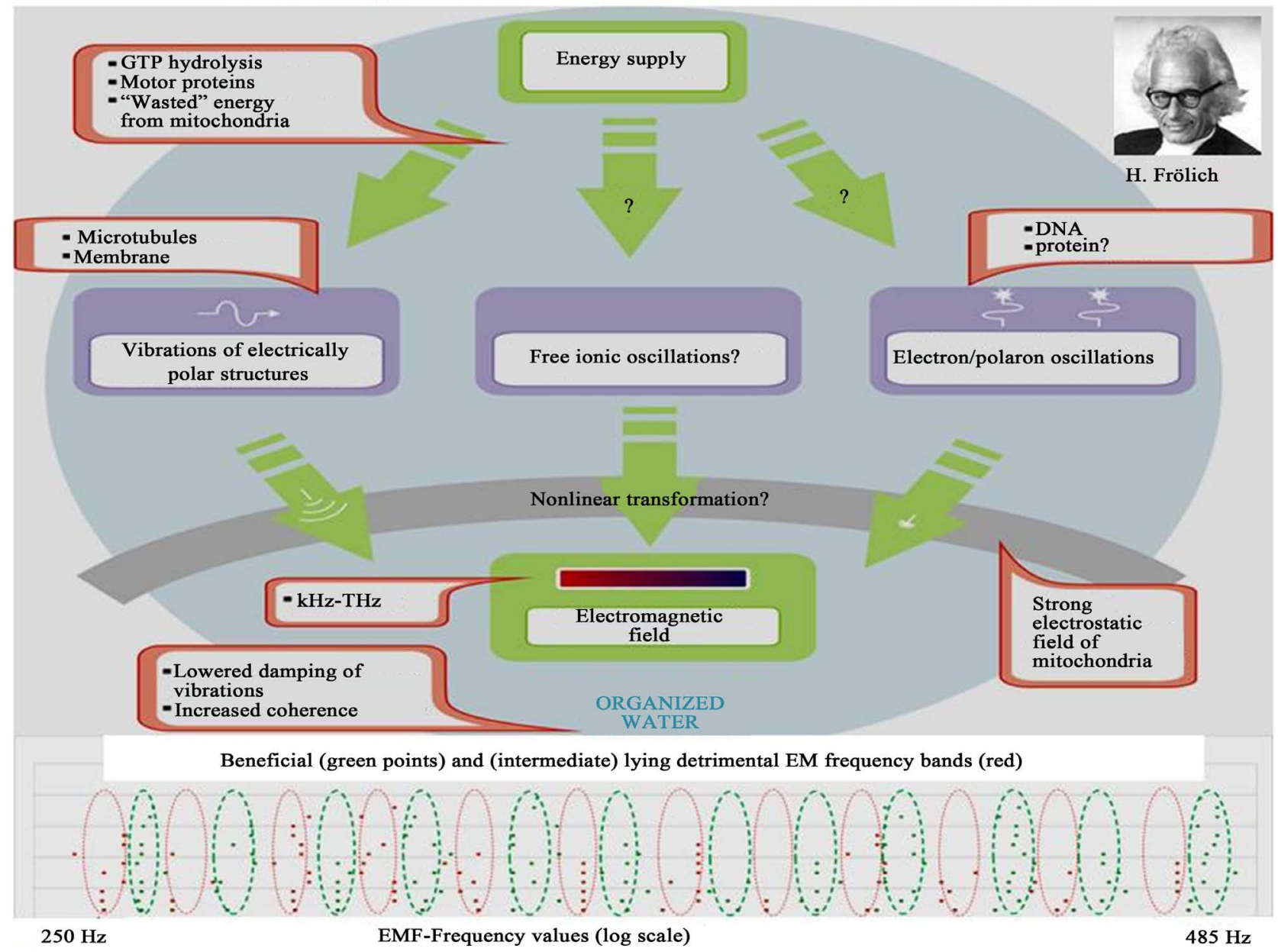

Figure 9. The EM resonance aspect of the integrate cell (electrome) with oscillating constituents at various intracellular sites (green arrows). Bottom part: EM field frequency band pattern of coherent (green points) and non-coherent values, as revealed in a meta-analyses of biomedical literature (see Geesink and Meijer, ref, nr 10, 11, 12). 
directed at the morphogenetic aspects of biological evolution. This also implies the requirement of a field receptive memory structure that can faithfully collect and store the integral history and present state of the particular cell, would be required.

In this respect, a toroidal space-time unit (hypersphere), encompassing a fourth spatial dimension and time symmetry is proposed. This represents an integral and updated holographic endo-memory of the exo-world that is instrumental in guiding the required life-architecture of cell structure and conformation of macromolecules to an optimal functional state for survival. For the latter aspect, potential retro-causality, through probing future viable states may be necessary. In this manner crucial a-priory information for the unfolding of life processes can be detected and the final function of the protein in the cellular protein networks can be anticipated and assigned

In conclusion: proteins in life-systems indeed are never alone: they are guest in a multi-layered energy landscape, yet faithfully coupled with a memory modality. This supervening information can coordinate the fine tuning through constant feed-back and quality control on the basis of the representation of a versatile network of force fields that interact with those of the cell. Toroidal geometry of discrete electromagnetic wave frequency patterns, reflecting soliton (polaron) energies at the fractal scales of life systems is proposed to be instrumental in this respect. Distinct life information can be derived from the all pervading zero-point energy field, that is seen as a stochastic electro-dynamic system, but partly functions as a strongly correlated information matrix with dominating collective effects that can drive coherence and attractor states in cell components. Such a piloting steering system seems to provide a deterministic aspect to quantum mechanics [12], yet it should be realized that this kind of quantum transfer includes a back-reaction into the vacuum field and is a symmetric process [143], by which the field is continuously updated. This implies that the information flow cannot be fully deterministic, since the overall background is never the same.

We stipulate in the present paper that the recognition of resonant frequencies can lead to modulation of form and function of proteins and DNA, but that, associated with each cell, an integrated information processing center is required for an orchestrated effort to maintain cell viability and proper intercellular communication. It remains to be established whether a collective cellular memory workspace of the organism contributes to epigenetic perturbations and/or organ- and total body-memory. In this sense such a holographic memory space, as described by us for the human brain [97] may, in certain special stages of tissue development and regeneration, supplement known transcriptional mechanisms [144] and be instrumental in encoding distinct life information of non-neural nature.

The semi-harmonic GM-scale, of EM fields that influence a wide spectrum of oscillating systems in nature (see Figure 9), as revealed by us, may therefore 
represent a generalized biophysical principle with a "musical" signature that provides an interplay of electromagnetic waves, highlighting the electro-tonal features that underlies life and the fabric of reality in general. It is of interest that Niemetz [145] reviewed the recent findings of micro-musical aspects of whole cells, DNA and proteins by atomic force microscopy ("tactoscope"), also mentioning the scale invariant recorded, discrete, sounds of in-animated physical structures, up to black holes in the universe. All this points at a deeper layer of a harmonically guided evolution [146], as was also revealed by Frank Wilczek, in his book “A Beautiful Question, on Finding Nature's Deep Design” [147].

\section{References}

[1] Melkikh, A.V. and Meijer, D.K.F. (2018) On a Generalized Levinthal's Paradox: The Role of Long- and Short Range Interactions on Complex Bio-Molecular Reactions, including Protein and DNA Folding. Progress in Biophysics and Molecular Biology, 132, 57-79. https://doi.org/10.1016/j.pbiomolbio.2017.09.018

[2] Bédard, S., Krishna, M.M.G., Mayne, L. and Englander, W.S. (2008) Protein Folding: Independent Unrelated Pathways or Predetermined Pathway with Optional Errors. PNAS, 105, 7182-7187. https://doi.org/10.1073/pnas.0801864105

[3] Martinez, L. (2014) Introducing the Levinthal's Protein Folding Paradox and Its Solution. Journal of Chemical Education, 91, 1918-1923. https://doi.org/10.1021/ed300302h

[4] Chen, M., Lin, X., Zheng, W., Onuchic, J.N. and Wolynes, P.G. (2016) Protein Folding and Structure Prediction from the Ground Up: The Atomistic Associative Memory, Water Mediated, Structure and Energy Model. The Journal of Physical Chemistry B, 120, 8557-8565. https://doi.org/10.1021/acs.jpcb.6b02451

[5] Wolynes, P.G. (2015) Evolution, Energy Landscapes and the Paradoxes of Protein Folding. Biochimie, 119, 218-230. https://doi.org/10.1016/j.biochi.2014.12.007

[6] Muñoz, V. and Cerminara, M. (2016) When Fast Is Better: Protein Folding Fundamentals and Mechanisms from Ultrafast Approaches. Biochemical Journal, 473, 2545-2559. https://doi.org/10.1042/BCJ20160107

[7] De Loof, A. (2016) The Cell's Self-Generated "Electrome”: The Biophysical Essence of the Immaterial Dimension of Life? Communicative \& Integrative Biology, 9, e1197446. https://www.researchgate.net/publication/304712940 https://doi.org/10.1080/19420889.2016.1197446

[8] Tamulis, A. (2008) Quantum Mechanical Control of Artificial Minimal Living Cells. NeuroQuantology, 6, 311-322. https://doi.org/10.14704/nq.2008.6.3.188

[9] Tamulis, A. and Tamulis, V. (2008) Quantum Mechanical Design of Molecular Electronics OR Gate for Regulation of Minimal Cell Functions. Journal of Computational and Theoretical Nanoscience, 5, 545-553. https://doi.org/10.1166/jctn.2008.2497

[10] Geesink, J.H. and Meijer, D.K.F. (2016) Quantum Wave Information of Life Revealed: An algorithm for EM Frequencies That Create Stability of Biological Order, with Implications for Brain Function and Consciousness. NeuroQuantology, 14, 106-125. https://doi.org/10.14704/nq.2016.14.1.911

[11] Geesink, J.H. and Meijer, D.K.F. (2016) Electromagnetic Frequency Patterns That Are Crucial for Health and Disease Reveal a Generalized Biophysical Principle: The GM Scale. Quantum Biosystems, 8, 1-16. 
[12] Geesink, J.H. and Meijer, D.K.F. (2018) Structure for Electromagnetic Frequencies That May Reflect Pilot Waves of Bohm's Implicate Order. Journal of Modern Physics, 9, 851-897. http://www.scirp.org/journal/jmp https://doi.org/10.4236/jmp.2018.95055

[13] Belyaev, I. (2015) Biophysical Mechanisms for Nonthermal Microwave Effects. In: Markov, M.S., Ed., Electromagnetic Fields in Biology and Medicine, CRC Press, Boca Raton, 49-68. https://doi.org/10.1201/b18148-6

[14] Brizhik, L. (2013) Biological Effects of Pulsating Magnetic Fields: Role of Solitons.

[15] Cifra, M., Fields, J.Z. and Farhadi, A. (2011) Electromagnetic Cellular Interactions. Progress in Biophysics and Molecular Biology, 105, 223-246.

[16] Cosic, I., Cosic, D. and Lazar, K. (2015) Is It Possible to Predict Electromagnetic Resonances in Proteins, DNA and RNA? Nonlinear Biomedical Physics, 3, 5.

[17] Hammerschlag, R., Levin, M., Mc Craty, R., Bat, B.A., Ives, J.A., Lutgendorf, S.K. and Oschman, J.L. (2015) Biofield Physiology: A Framework for an Emerging Discipline. Global Advances in Health and Medicine, 4, 35-41.

[18] Levin, M. (2012) Molecular Bioelectricity in Developmental Biology: New Tools and Recent Discoveries: Control of Cell Behavior and Pattern Formation by Transmembrane Potential Gradients. BioEssays, 34, 205-217.

https://doi.org/10.1002/bies.201100136

[19] Muehsam, D. and Ventura, C. (2014) Life Rhythm as a Symphony of Oscillatory Patterns: Electromagnetic Energy and Sound Vibration Modulates Gene Expression for Biological Signaling and Healing. Global Advances in Health and Medicine, 3, 40-55. https://doi.org/10.7453/gahmj.2014.008

[20] Pokorny, J. (2004) Excitation of Vibrations in Microtubules in Living Cell. Bioelectrochemistry, 63, 321-326. https://doi.org/10.1016/j.bioelechem.2003.09.028

[21] Rakovic, D., Dugic, M. and Cirkovic, M.M. (2004) Macroscopic Effects in Biophysics and Consciousness. NeuroQuantology, 2, 237-262.

[22] Tuszynski, J.A., Wenger, C., Friesen, D.E. and Preto, J. (2016) An Overview of Sub-Cellular Mechanisms Involved in the Action of TTFields. International Journal of Environmental Research and Public Health, 13, 1128. https://doi.org/10.1016/j.bioelechem.2003.09.028

[23] Redozubov, A. (2017) Holographic Memory: A Novel Model of Information Processing by Neuronal Microcircuits. In: Opris, Ioan, Casanova and Manuel, F., Eds., The Physics of the Mind and Brain Disorders, Springer Series in Cognitive and Neural Systems Chapter 13, Springer, Berlin, 271-295.

[24] Preto, J. (2016) Classical Investigation of Long Range Coherence in Biological Systems. Chaos, 26, Article ID: 123116. https://doi.org/10.1063/1.4971963

[25] Reimers, J.R., McKemmish, L.K., McKenzie, R.H., Mark, A.E. and Hush, N.S. (2009) Weak, Strong, and Coherent Regimes of Fröhlich Condensation and Their Applications to Terahertz Medicine and Quantum Consciousness. PNAS, 106, 4219-4224. https://doi.org/10.1073/pnas.0806273106

[26] Lundholm, I.V., Rodilla, H., Wahlgren, W.Y., Duelli, A., Bourenkov, G., Vukusic, J., Friedman, R., Stake, J., Schneider, T. and Katona, G. (2015) Terahertz Radiation Induces Non-Thermal Structural Changes Associated with Fröhlich Condensation in a Protein Crystal. Structural Dynamics, 2, Article ID: 054702. https://doi.org/10.1063/1.4931825

[27] Nardecchia, I., Torres, J., Lechelon, M., Giliberti, V., Ortolani, M., Nouvel, P., Gori, M., Donato, I., Preto, J., Varani, L., Sturgis, J. and Pettini, M. (2017) Out-of-Equilibrium 
Collective Oscillation as Phonon Condensation in a Model Protein. https://arxiv.org/pdf/1705.07975.pdf

[28] Nardecchia, I., Spinelli, L., Preto, J., Gori, M., Floriani, E., Jaeger, S., Ferrier, P. and Pettini, M. (2014) Experimental Detection of Long-Distance Interactions between Biomolecules through Their Diffusion Behavior: Numerical Study. Physical Review E, 90, Article ID: 022703. https://doi.org/10.1103/PhysRevE.90.022703

[29] Goushcha, A.O., Hushcha, T.O. and Christophorov, L.N. (2014) Self-Organisation and Coherence in Biology and Medicine. Open Journal of Biophysics, 4, 119-146. https://doi.org/10.4236/ojbiphy.2014.44014

[30] Rost, B. (2003) Neural Network Predict Protein Structure: Hype or Hit? In: Frasconi, P. and Shamir, Eds., Artificial Intelligence and Heuristic Methods in Bioinformatics, IOS press, Amsterdam, 34-50.

[31] Fröhlich, H. (1975) The Extraordinary Dielectric Properties of Biological Materials and the Action of Enzymes. PNAS, 72, 4211-4215. https://doi.org/10.1073/pnas.72.11.4211

[32] Davydov, A.S. (1977) Solitons and Energy Transfer along Protein Molecules. Journal of Theoretical Biology, 66, 379-387. https://doi.org/10.1016/0022-5193(77)90178-3

[33] Davydov, A.S. (1973) The Theory of Contraction of Proteins under Their Excitation. Journal of Theoretical Biology, 38, 559-569. https://doi.org/10.1016/0022-5193(73)90256-7

[34] Pang, X.F., Chen, S., Wang, X. and Zhong, L. (2016) Influences of Electromagnetic Energy on Bio-Energy Transport through Protein Molecules in Living Systems and Its Experimental Evidence. International Journal of Molecular Sciences, 17, 1130. https://doi.org/10.3390/ijms17081130

[35] Cruzeiro, L. (2009) The Davydov/Scott Model for Energy Storage and Transport in Proteins. Journal of Biological Physics, 35, 43-55. https://www.ncbi.nlm.nih.gov/pmc/articles/PMC2660397

[36] Ritz, W. (1909) Theorie der Transversalschwingungen einer quadratischen Platte mit freien Rändern. Annalen der Physik, 333, 737-786.

[37] Chladni, E.F.F. (1817) Neue Beyträge zur Akustik, by Ernst Florens Friedrich Chladni. Breitkopf und Härtel, Leipzig.

[38] Geesink, J.H. and Meijer, D.K.F. (2017) Bio-Soliton Model That Predicts Non-Thermal Electromagnetic Frequency Bands, That Either Stabilize or Destabilize Living Cells. Electromagnetic Biology and Medicine, 36, 357-378. https://doi.org/10.1080/15368378.2017.1389752

[39] Salford, L.G., Nitty, H., et al. (2017) The Mammalian Brain in the Electromagnetic Fields Designed by Man with Special Reference to Blood-Brain Barrier Function, Neuronal Damage and Possible Physical Mechanisms. Progress of Theoretical Physics Supplement, 173, 283-309. http://ptp.ipap.jp/link?PTPS/173/283

[40] Tozzi, A. and Peters, J. (2017) Plasma-Like Brain: Collective Movements in the Extracellular Nervous Spaces.

https://www.researchgate.net/publication/304777768_PLASMALIKE_BRAIN_COL LECTIVE_MOVEMENTS_IN_THE_EXTRACELLULAR_NERVOUS_SPACES

[41] Sahu, S., Ghosh, S., Ghosh, B., Aswani, K., Hirata, K., Fujita D., et al. (2013) Atomic Water Channel Controlling Remarkable Properties of a Single Brain Microtubule: Correlating Single Protein to Its Supramolecular Assembly. Biosensors and Bioelectronics, 47, 141-148. https://doi.org/10.1016/j.bios.2013.02.050 
[42] Zioutas, K. (1996) On the Interaction of Extreme-Low-Frequency (ELF) Radiation with Living Matter's Coherent Spiral States. https://arxiv.org/pdf/patt-sol/9601001

[43] Cheron, G., Gall, D., Servais, L., Dan, B., Maex, R. and Schiffmann, S.N. (2004) Inactivation of Calcium-Binding Protein Genes Induces $160 \mathrm{~Hz}$; Oscillations in the Cerebellar Cortex of Alert Mice. The Journal of Neuroscience, 24, 434-441. https://doi.org/10.1523/JNEUROSCI.3197-03.2004

[44] Persinger, M.A., Murugan, N.J. and Karbowski, L.M. (2015) Combined Spectral Resonances of Signaling Proteins' Amino Acids in the ERK-MAP Pathway Reflect Unique Patterns That Predict Peak Photon Emissions and Universal Energies. International Letters of Chemistry, Physics and Astronomy, 43, 10-25. https://doi.org/10.18052/www.scipress.com/ILCPA.43.10

[45] Sahu, S., Ghosh, S., Fujita, D. and Bandyopadhyay, A. (2014) Live Visualizations of single Isolated Tubulin Protein Self-Assembly via Tunneling Current: Effect of Electromagnetic Pumping during Spontaneous Growth of Microtubule. Scientific Reports, 4, Article No. 07303. https://doi.org/10.1038/srep07303

[46] Ghosh, S., Sahu, S., Agrawal, L., Shiga, T. and Bandyopadhyay, A. (2016) Inventing a Co-Axial Atomic Resolution Patch Clamp to Study a Single Resonating Protein Complex and Ultra-Low Power Communication Deep inside a Living Neuron Cell. Journal of Integrative Neuroscience, 15, 403-433. https://doi.org/10.1142/S0219635216500321

[47] Copty, A.B., Neve-Oz, Y., Barak, I., Golosovsky, M. and Davidov, D. (2006) Evidence for a Specific Microwave Radiation Effect on the Green Fluorescent Protein. Biophysical Journal, 91, 1413-1423. https://doi.org/10.1529/biophysj.106.084111

[48] Meijer, D.K.F. and Geesink, J.H. (2016) Phonon Guided Biology: Architecture of Life and Conscious Perception Are Mediated by Toroidal Coupling of Phonon, Photon and Electron Information Fluxes at Discrete Eigenfrequencies. NeuroQuantology, 14, 718-755. https://doi.org/10.14704/nq.2016.14.4.985

[49] Gerner, C., Haudek, V., Schandl, U., Bayer, E., Gundacker, N., Hutter, H.P. and Mosgoeller, W. (2010) Increased Protein Synthesis by Cells Exposed to a $1,800-\mathrm{MHz}$ Radio-Frequency Mobile Phone Electromagnetic Field, Detected by Proteome Profiling. Archives of Occupational and Environmental Health, 83, 691-702. https://doi.org/10.1007/s00420-010-0513-7

[50] Cheon, H., Yang, H.J., Lee S.H., Kim, Y.A. and Son, J.H. (2016) Terahertz Molecular Resonance of Cancer DNA. Scientific Reports, 6, Article No. 37103. https://doi.org/10.1038/srep37103

[51] Geesink, J.H. and Meijer, D.K.F. (2018) A Semi-Harmonic Frequency Pattern Organizes Local and Non-Local States by Quantum Entanglement in both EPR-Studies and Life Systems. Journal of Modern Physics, 9, 898-924.

http://www.scirp.org/journal/jmp https://doi.org/10.4236/jmp.2018.95056

[52] Geesink, J.H. and Meijer, D.K.F. (2018) Semi-Harmonic Scaling Enables Calculation of Masses of Elementary Particles of the Standard Model. Journal of Modern Physics, 9, 925-947. http://www.scirp.org/journal/jmp https://doi.org/10.4236/jmp.2018.95057

[53] Adamatzky, A. (2013) Game of Life on Phyllosilicates: Gliders, Oscillators and Still Life. Physics Letters, 377, 597-1605.

[54] Hashizume, H. (2012) Role of Clay Minerals in Chemical Evolution and the Origins of Life. http://uu.diva-portal.org/smash/get/diva2:935529/FULLTEXT01.pdf 
[55] Grandpierre, A. (2001) Phenomenology of Space and Time. Book 1. The Forces of the Cosmos and the Ontopoietic Genesis of Life. In: Tymieniecka, A.-T. Ed., Analecta Husserliana. http://www.springer.com/philosophy/book/978-3-319-02014-3

[56] Meijer, D.K.F. (2012) The Information Universe. On the Missing Link in Concepts on the Architecture of Reality. Syntropy Journal, 1, 1-64.

[57] Walker, S.I. and Davies, P.C.W. (2013) The Algorithmic Origin of Life. Journal of the Royal Society Interface, arXiv:1207.4803. https://doi.org/10.1098/rsif.2012.0869

[58] Dotta, B.T., Mulligan, B.P., Hunter, M.D. and Persinger, M.A. (2009) Evidence of Macroscopic Quantum Entanglement during Double Quantitative Electroencephalographic Measurements of Friend's vs Strangers. NeuroQuantology, 7, 548-551. https://doi.org/10.14704/nq.2009.7.4.251

[59] Melkikh, A.V. (2014) Quantum Information and the Problem of Mechanisms of Biological Evolution. BioSystems, 115, 33-45. https://doi.org/10.1016/j.biosystems.2013.10.005

[60] Melkikh, A.V. and Mahecha, D.S. (2018) On the Broader Sense of Life and Evolution: Its Mechanisms, Origin and Probability across the Universe. Journal of Astrobiology and Outreach, 6, 1-13.

[61] Tuszynski, M.H., et al. (2015) Nerve Growth Factor Gene Therapy: Activation of Neuronal Responses in Alzheimer Disease. JAMA Neurology, 72, 1139-1147. https://doi.org/10.1001/jamaneurol.2015.1807

[62] Hameroff, S. and Penrose, R. (2014) Consciousness in the Universe: A Review of the “Orch OR" Theory. Physics of Life Reviews, 11, 39-78. https://doi.org/10.1016/j.plrev.2013.08.002

[63] Craddock, T.J.A., Friesen, D., Mane, J., Hameroff, S. and Tuszynski, J. (2014) The Feasibility of Coherent Energy Transfer in Microtubules. Journal of the Royal Society Interface, 11, Article ID: 20140677.

[64] Foletti, A. and Brizhik, L. (2017) Non-Linearity Coherence and Complexity: Biological Aspects Related to Health and Disease. Electromagnetic Biology and Medicine, 36, 315-324. https://doi.org/10.1080/15368378.2017.1371034

[65] Laurell, H. (2016) A Summary on Solitons in Quantum Field Theory. http://uu.diva-portal.org/smash/get/diva2:935529/FULLTEXT01.pdf

[66] Molkenthin, N., Hu, S. and Niemi, A.J. (2010) Discrete Nonlinear Schrodinger Equation, Solitons and Organizing Principles for Protein Folding. https://arxiv.org/abs/1009.1078

[67] Halgamuge, M.N., Perssont, B.R.R. and Salford, G. (2009) Comparison between Two Models for Interactions between Electric and Magnetic Fields and Proteins in Cell Membranes. Environmental Engineering Science, 26, 1473-1480. https://doi.org/10.1089/ees.2009.0014

[68] Chernodub, M., Hu, S.W. and Niemi, A.J. (2010) Topological Solitons and Folded Proteins. Physical Review E, 82, Article ID: 011916. https://doi.org/10.1103/PhysRevE.82.011916

[69] Jerman, I. (2016) The Origin of Life from Quantum Vacuum, Water and Polar Molecules. American Journal of Modern Physics, 5, 34-43.

[70] Henry, M. (2016) Hofmeister Series: The Quantum Mechanical Viewpoint. Current Opinion in Colloid \& Interface Science; 23, 119-125. https://doi.org/10.1016/j.cocis.2016.08.001

[71] Levy, Y. and Onuchie J.N. (2006) Water Mediation in Protein Folding and Molecular Recognition. Annual Review of Biophysics and Biomolecular Structure, 35, 
389-415. https://doi.org/10.1146/annurev.biophys.35.040405.102134

[72] Chaplin, M.F. (2000) A Proposal for the Structuring of Water. Biophysical Chemistry, 83, 211-221. https://doi.org/10.1016/S0301-4622(99)00142-8

[73] Johnson, K. (2009) "Water Buckyball” Terahertz Vibrations in Physics, Chemistry, Biology, and Cosmology. https://arxiv.org/ftp/arxiv/papers/0902/0902.2035.pdf

[74] Tielrooij, K.J., Garcia-Araez, N., Bonn, M. and Bakker, H.J. (2010) Cooperativity in Ion Hydration. Science, 328, 1006-1009. https://doi.org/10.1126/science.1183512

[75] Chai, B., Yoo, H. and Pollack, G.H. (2009) Effect of Radiant on Near-Surface Water. The Journal of Physical Chemistry B, 113, 13953-13958. https://doi.org/10.1021/jp908163w

[76] Carniello, T.N., Vares, D.A.E. and Persinger, M.A. (2015) Quantitative Support for Water as the Conduit of Interaction for Universal Entanglement. Journal of Consciousness Exploration \& Research, 6, 738-749.

[77] Havenith, M. (2010) Water and Biological Molecules Probed by Terahertz-Spectroscopy. http://www.hfsp.org/frontier-science/hfsp-success-stories/water-and-biological-mol ecules-probed-terahertz-spectroscopy

[78] Ball, P. (2017) Water Is an Active Matrix of Life for Cell and Molecular Biology. PNAS, 114, 13327-13335. https://doi.org/10.1073/pnas.1703781114

[79] Sbitnev, V.I. (2016) Quantum Consciousness in Warm, Wet, and Noisy Brain. Modern Physics Letters B, 30, 25 p. https://arxiv.org/abs/1606.00258 https://doi.org/10.1142/S0217984916503292

[80] Sbitnev, V.I. (2017) Hydrodynamics of Superfluid Quantum Space: De Broglie Interpretation of Quantum Mechanics.

[81] Mentré. P. (2012) Water in the Orchestration of the Cell Machinery. Some Misunderstandings: A Short Review. Journal of Biological Physics, 38, 13-26. https://doi.org/10.1007/s10867-011-9225-9

[82] Graziano, G. (2013) A View on the Dogma of Hydrophobic Imperialism in Protein Folding. Journal of Biomolecular Structure and Dynamics, 31, 1016-1019. https://doi.org/10.1080/07391102.2012.748545

[83] Montagnier, L., et al. (2015) Transduction of DNA Information through Water and Electromagnetic Waves. Electromagnetic Biology and Medicine, 34, 106-112. https://doi.org/10.3109/15368378.2015.1036072

[84] Montagnier, L., et al. (2017) Water Bridging Dynamics of Polymerase Chain Reaction in the Gauge Theory Paradigm of Quantum Fields. Water, 9, 339. https://doi.org/10.3390/w9050339

[85] Lawrence, D.S. and Shell, T.A. (2015) Vitamin $B_{12}$ : A Tunable, Long Wavelength, Light-Responsive Platform for Launching Therapeutic Agents. Accounts of Chemical Research, 48, 2866-2874. https://doi.org/10.1021/acs.accounts.5b00331

[86] Mc Dermott, M.L, Vanselous, H., Corcelli, S.A. and Petersen, P.B. (2017) DNA's Chiral Spine of Hydration. ACS Central Science, 3, 708-714. https://doi.org/10.1021/acscentsci.7b00100

[87] Plankar, M., Jerman, I. and Krasovec, R. (2011) On the Origin of Cancer: Can We Ignore Coherence? Progress in Biophysics and Molecular Biology, 106, 380-390. https://doi.org/10.1016/j.pbiomolbio.2011.04.001

[88] Todorova, N., Bentvelzen, A., English, N.J. and Yarovsky, I. (2016) Electromagnetic-Field Effects on Structure and Dynamics of Amyloidogenic Peptides. The Journal of Chemical Physics, 144, Article ID: 085101. https://doi.org/10.1063/1.4941108

[89] Pouthier, V. (2009) Narrow Band Excitation Coupled with Acoustical Anharmonic 
Phonons: Application to the Vibrational Energy Flow in Lattice of H-Bonded Peptide Units. Journal of Physics. Condensed Matter, 21, Article ID: 185404.

https://www.utinam.cnrs.fr/IMG/pdf/exciton.pdf https://doi.org/10.1088/0953-8984/21/18/185404

[90] Davies, P.C.W. (2014) Does Quantum Mechanics Play a Non-Trivial Role in Life? BioSystems, 78, 69-79. https://doi.org/10.1016/j.biosystems.2004.07.001

[91] Farnsworth, K.D., Nelson, J. and Gershenson, C. (2013) Living Is Information Processing: From Molecules to Global Systems. Acta Biotheoretica, 61, 203-222. https://doi.org/10.1007/s10441-013-9179-3

[92] Pribram, K.H. (2004) Consciousness Reassessed. Mind and Matter, 2, 7-35.

[93] Bieberich, E. (2012) Introduction in Fractality Principle of Consciousness and Sentyon Postulate. Cognitive Computation, 4, 13-28. https://doi.org/10.1007/s12559-011-9104-5

[94] Susskind, L. (1994) The World as a Hologram.

[95] 't Hooft, G. (2001) The Holographic Principle. In: Zuchichi, A., Ed., Basics and Highlights in Fundamental Physics, World Scientific, Singapore, 72-100. https://doi.org/10.1142/9789812811585_0005

[96] Bieberich, E. (2014) Synthesis, Processing, and Function of N-Glycans in N-Glycoproteins. Glycobiology of the Nervous System, 9, 47-70. https://doi.org/10.1007/978-1-4939-1154-7_3

[97] Meijer, D.K.F. and Geesink, J.H. (2017) Consciousness in the Universe Is Scale Invariant and Implies an Event Horizon of the Human Brain. NeuroQuantology, 15, 41-79.

[98] Anjamrooz, S.H. (2013) The Cellular Memory Disc of Reprogrammed Cells. Stem Cell Reviews and Reports, 9, 190-209. https://doi.org/10.1007/s12015-013-9429-4

[99] Anjamrooz, S.H. (2015) Cell Memory-Based Therapy. Journal of Cellular and Molecular Medicine, 19, 2682-2689. https://doi.org/10.1111/jcmm.12646

[100] Hales, C.G. (2014) The Origin of Brain's Endogenous Electromagnetic Field and Its Relation with Consciousness. Journal of Integrative Neuroscience, 13, 313-361. https://doi.org/10.1142/S0219635214400056

[101] Bernroider, G. (2003) Quantum Neurodynamics and the Relationship to Conscious Experience. NeuroQuantology, 1, 163-168.

[102] Cifra, M., Pokorny, J., Havelka, D. and Kucer, O. (2010) Electric Field Generated by Axial Longitudinal Vibration Modes of Microtubule, BioSystems, 100, 122-131. https://doi.org/10.1016/j.biosystems.2010.02.007

[103] Farhadi, A., Forsyth, C., Banan, A., Shaikh M., Engen, P., Fields, J.Z. and Keshavarzian, A. (2007) Evidence for Non-Chemical, Non-Electrical Intercellular Signaling in Intestinal Epithelial Cells. Bioelectrochemistry, 71, 142-148. https://doi.org/10.1016/j.bioelechem.2007.03.001

[104] Persinger, M.A. and Dotta, B.T. (2011) Temporal Patterns of Photon Emission Can Be Stored and Retrieved Several Days Later from the "Same Space": Experimental and Quantitative Evidence. NeuroQuantology, 9, 605-613. https://doi.org/10.14704/nq.2011.9.4.467

[105] Gough, W.C. and Shacklett R.L. (1993) "Physics, Parapsychology and Religion"-Part I: The Reality Beyond Space-Time, Part II: The Quantum Linkage, Part III: The Human Implications. Journal of Religion and Psychical Research, 16, 65-77, 126-134, 196-209. 
[106] Levin, M.A. and Wen, X.-G. (2005) String-Net Condensation: A Physical Mechanism for Topological Phases. Physical Review B, 71, Article ID: 045110

[107] Arkani-Hamed, N. (2013) Beyond the Standard Model Theory. Physica Scripta, 2013, T158.

[108] Merali, Z. (2017) The Universe Is a String-Net Liquid. http://dao.mit.edu/ wen/NSart-wen.html

[109] Tegmark, M. (2014) Our Mathematical Universe. My Quest for the Ultimate Nature of Reality. Penquin Books Ltd., London.

[110] Butterfield, J. (2014) Our Mathematical Universe. Book Review in Plus Magazine of the UK Mathematics Millenium Project.

[111] Azvedo, E. and Filho, J.P. (2017) Is There an Information Field in the Life World? Empirical Approach Using Electrophotonic Analysis. Journal of Life Sciences, 11, 191-201.

[112] Fleming, A.H.J. (2017) A Range of Fields over the Spectrum in a Cell Colony May Control the Timing of Its Cell Cycle. 2017 Progress in Electromagnetics Research Symposium-Spring (PIERS), St. Petersburg, 22-25 May 2017. https://doi.org/10.1109/PIERS.2017.8262346

[113] Tozzi, A. and Peters, J.F. (2016) Towards a Fourth Spatial Dimension of Brain Activity. Cognitive Neurodynamics, 10, 189-199. https://doi.org/10.1007/s11571-016-9379-Z

[114] Haramein, N, Brown, W.D. and Val Baker, A. (2016) The United Space Memory Network: From Cosmogenesis to Consciousness. NeuroQuantology, 14, 1-15. https://doi.org/10.14704/nq.2016.14.4.961

[115] Geesink, J.H. and Meijer, D.K.F. (2017) Bio-Soliton Model That Predicts Non-Thermal Electromagnetic Frequency Bands, That Either Stabilize or Destabilize Living Cells. Electromagnetic Biology and Medicine, 36, 357-378. https://doi.org/10.1080/15368378.2017.1389752

[116] Rouleau, N. and Dotta, B.T. (2014) Electromagnetic Fields as Structure Function Zeitgebers in Biological Systems: Environmental Orchestration and Consciousness. Frontiers in Integrative Neuroscience, 8, 84.

[117] Salari, V., Vallian, H., Bassereh, H., Bókkon, I. and Barhordari, A. (2015) Ultraweak Photon Emission in the Brain. Journal of Integrative Neuroscience, 14, 1-11. https://doi.org/10.1142/S0219635215300012

[118] Laager, F. (2015) Light Based Cellular Interactions: Hypotheses and Perspectives. Frontiers in Physics, 3, 55. https://doi.org/10.3389/fphy.2015.00055

[119] Mayburov, S.N. (2012) Photonic Communication and Information Encoding in Biological Systems. arXiv:1205.4134.

[120] Tang, R. and Dai, J. (2014) Spatiotemporal Imaging of Glutamate-Induced Biophotonic Activities and Transmissions in Neural Circuits. PLOS ONE, 9, e85643. https://doi.org/10.1371/journal.pone.0085643

[121] Wang, Z., Wang, N., Li, Z., Xiao, F. and Dai, J. (2016) Human High Intelligence Is Involved in Spectral Redshift of Biophotonic Activities in the Brain. PNAS, 113, 8753-8758. https://doi.org/10.1073/pnas.1604855113

[122] Meijer, D.K.F. (2014) The Extended Brain: Cyclic Information Flow in a Quantum Physical Realm. NeuroQuantology, 12, 180-200.

[123] Penrose, R. (2014) On the Gravitization of Quantum Mechanics 1: Quantum State Reduction. Foundations of Physics, 44, 557-575. https://doi.org/10.1007/s10701-013-9770-0 
[124] Edwards, J.C.W. (2015) Is Consciousness only a Property of Individual Cells? Journal of Consciousness Studies, 12, 60-76.

[125] Edwards, J.C.W. (2016) Distinguishing Representations as Origin and as Input: Roles for Individual Neurons. Frontiers in Psychology, 7, 1537. https://doi.org/10.3389/fpsyg.2016.01537

[126] Sevush, S. (2004) Single-Neuron Theory of Consciousness. https://www.ncbi.nlm.nih.gov/pubmed/16083912 http://cogprints.org/3891/1/snt-9html.htm

[127] Lisi, A., Ledda, M., De Carlo, F., Foletti, A., Giuliani, L., D’Emilia, E. and Grimaldi, S. (2008) Calcium ion Cyclotron Resonance (ICR) Transfers Information to Living Systems: Effects on Human Epithelial Cell Differentiation. Electromagnetic Biology and Medicine, 27, 230-240. https://doi.org/10.1080/15368370802269135

[128] Huelga, S.F. and Plenio, M.B. (2013) Vibartion, Quanta and Biology. Contemporary Physics, 54, 181-207.

[129] Stoop, R., Buchli, J., Keller, G. and Steeb, W.-H. (2003) Stochastic Resonance in Pattern Recognition by a Holographic Neuron Model. Physical Review E, 67, Article ID: 061918. https://doi.org/10.1103/PhysRevE.67.061918

[130] Pereira, A. and Furlan, F.A. (2007) Biomolecular Information, Brain Activity and Cognitive Function. Annual Review of Biomedical Sciences, 9, 12-29. https://doi.org/10.3389/fpsyg.2013.00200

[131] Sutherland, J.G. (1994) The Holographic Cell: A Quantum Perspective. In: Plantamura, V.L., et al., Eds., Frontier Decision Support Concepts, John Wiley \& Sons, New York.

[132] Baars, B.J., Franklin, S. and Zoege Ramsoy, T. (2013) Global Workspace Dynamics: Cortical "Binding and Propagation" Enables Conscious Contents. Frontiers in Psychology, 4, 1-22.

[133] Fröhlich, F. and McCormick, D.A. (2010) Endogenous Electric Fields May Guide Neocortical Network Activity. Neuron, 67, 129-143. https://doi.org/10.1016/j.neuron.2010.06.005

[134] Keppler, J.A. (2013) A New Perspective on the Functioning of the Brain and the Mechanisms behind Conscious Processes. Frontiers in Psychology, 4, 242.

[135] Persinger, M.A. and Lavallee, C.F. (2010) Theoretical and Experimental Evidence of Macroscopic Entanglement between Human Brain Activity and Photon Emissions: Implications for Quantum Consciousness and Future Applications. Journal of Consciousness Exploration \& Research, 1, 785-807.

[136] Gabella, M. (2006) The Randall-Sundrum Model.

[137] Auletta, G., Ellis, G.F.R. and Jaeger, L. (2008) Top-Down Causation by Information Control: From Philosophical Problem to Scientific Research Program. Journal of the Royal Society Interface, 5, 1159-1172. https://doi.org/10.1098/rsif.2008.0018

[138] Perus. M. and Bishof, H. (2003) A Neural-Network Quantum Information Processing System. Neural Network World, 10, 1001-1013.

[139] Ezhov, A.A. and Ventura, D. (2000) Quantum Neural Networks. In: Nikola, K., Ed., Future Directions for Intelligent Systems and Information Sciences, 213-235.

[140] Rakovic, D., Dugic, M., Jeknic-Dugic, M., Plavicik, M., Jacimovski, S. and Setracic, J. (2014) On Macroscopic Quantum Phenomena in Biomolecules and Cells: From Levinthal to Hopfield. BioMed Research International, 2014, Article ID: 580491.

https://www.hindawi.com/journals/bmri/2014/580491

https://doi.org/10.1155/2014/580491 
[141] Ling, G.N. (2014) Can We See Living Structures in a Cell. Physiological Chemistry and Physics and Medical NMR, 43, 1-71.

[142] Jaeken, L. (2017) The Neglected Functions of Intrinsically Disordered Proteins and the Origin of Life. Progress in Biophysics and Molecular Biology, 126, 31-46. https://doi.org/10.1016/j.pbiomolbio.2017.03.002

[143] Sutherland, R.I. (2006) Causally Symmetric Bohm Model. https://pdfs.semanticscholar.org/c5f0/8198ef854f5fd824110d9aab05101879d63c.pdf

[144] Burrill D.R. and Silver, P.A. (2010) Making Cellular Memories. Cell, 140, 13-18. https://doi.org/10.1016/j.cell.2009.12.034

[145] Niemetz, A. (2004) Singing Cells, Art, Science and Noise in between. MFA Thesis, UCLA Department of Design/Media Arts, University of California, Los Angeles.

[146] Merrick, R. (2010) Harmonically Guided Evolution. Fairview.

[147] Wilczek, F. (2016) A Beautiful Question, Finding Nature’s Deep Design. Penquin Books, London. 